\title{
COMPARACIÓN DE ESTRUCTURAS RÍGIDAS CON ESTRUCTURAS FLEXIBLES SOBRE AISLADORES SÍSMICOS FPT
}

\section{COMPARING RIGID AND FLEXIBLE STRUCTURES ON TRIPLE FRICTION PENDULUM (TFP) SEISMIC ISOLATORS}

\author{
Gissela Villarruel, Roberto Aguiar \\ Departamento de Ciencias de la Tierra y la Construcción \\ Universidad de las Fuerzas Armadas ESPE \\ Av. Gral. Rumiñahui s/n. Valle de los Chillos, Ecuador \\ gevillarruel@espe.edu.ec \\ rraguiar@espe.edu.ec
}

\section{RESUMEN}

Se presenta el análisis sísmico, mediante los Métodos Espectral y Simplificado Lineal del ASCE 7-10, con una validación de resultados en el software estructural SAP2000 obteniendo una interfaz gráfica en 3D, aplicado al bloque estructural I ZE del nuevo edificio: "Vinculación con la Sociedad" de la Universidad de las Fuerzas Armadas ESPE - sede Santo Domingo, que tiene aisladores sísmicos de triple péndulo de fricción colocados sobre los plintos de cimentación.

Se realiza un análisis sísmico espacial, para obtener: periodos, desplazamientos, fuerzas laterales y derivas en el centro de masas de cada piso, tanto con el Método Espectral como el Simplificado Lineal, calculados con el sistema computacional CEINCI-LAB y el programa comercial SAP2000, ejecutados para dos alternativas de cálculo que son: modelo 1 (columnas más aisladores sísmicos FPT, denominada Estructura Flexible), y modelo 2 (muros de corte, columnas de hormigón armado y aisladores sísmicos FPT denominada Estructura Rígida).

Para ello en primer lugar se presenta el marco teórico del Método Simplificado Lineal, siguiendo lo prescrito en la norma de aislación Chile INN (2013) y en el $\boldsymbol{A S C E}$ 7-10, dónde considera que la superestructura va a desplazarse como un solo cuerpo rígido, es decir, trabaja como un sistema de un grado de libertad con un modelo constitutivo del aislador bilineal. Para el Método Espectral se trabajó con la curva establecida de tres fases, propuesta por Mc Vitty y Constantinou (2015) para el aislador sísmico de triple péndulo de fricción FPT. 
El análisis tanto para la Estructura Rígida como para la Flexible se lo realiza en los dos sentidos "X" y "Y", para dos espectros: el de diseño DBE y el máximo considerado MCE.

Finalmente, se comparan los resultados máximos probables para cada modelo calculado (Estructura Flexible y Estructura Rígida) con sus correspondientes cálculos estructurales según el Método Espectral, Simplificado Lineal y SAP2000; se observa cómo mejora considerablemente el comportamiento sismo resistente al tener Estructuras Rígidas de éste tipo.

Palabras Claves.- Aisladores sísmicos de triple péndulo de fricción (FPT). Sismo DBE y MCE. Método Espectral. Método Simplificado Lineal. SAP2000.

ABSTRACT

A seismic analysis is presented using the Linear Simplified Method and Spectral Element Method based on ASCE 7-10, the validation of results is carried out by the object-based structural software SAP2000 to obtain a 3D graphical interface which is applied to the structural block I ZE of the new building "Vinculación con la Sociedad" of "Universidad de las Fuerzas Armadas" ESPE in Santo Domingo. The building has Triple Friction Pendulum Bearing (TFPB) as a seismic isolator system on its foundations.

A spatial seismic analysis is performed to obtain periods, displacements, lateral forces and derivatives in the center of mass of each floor by using both the Spectral and Simplified Linear Methods calculated with the CEINCI-LAB computer system and SAP2000 commercial software; they are conducted to obtain two calculation alternatives: Model 1 (columns plus FPT seismic isolators called Flexible Structure), and Model 2 (shear walls, reinforced concrete columns called Rigid Structure).

The theoretical framework of the Simplified Linear Method is presented in order to carry out the analysis, following the seismic isolation standard Chile INN (2013) and ASCE 7-10 which state that a superstructure will be displaced as a single rigid body. In other words, it works as a system of one degree of freedom with a constitutive model of the bilinear isolator. As for the Spectral Method, it was performed with the established three-phase model proposed by McVitty and Constantinou (2015) for the triple- friction pendulum seismic isolator FPT.

Regarding the analysis for the Rigid and Flexible Structures, it is performed on " $X$ " and "Y" coordinates for obtaining two spectra: design basis earthquake (DBE) and maximum considered earthquake (MCE). 
Finally, the maximum probable results for each calculated model (Flexible Structure and Rigid Structure) are computed from their corresponding structural calculations according to the Spectral Element Method, Linear Simplified Method and SAP2000 structural software; it is observed that the seismic behavior is considerably improved when having this type of Rigid Structures.

Keywords: Triple Friction Pendulum (FPT) seismic isolator, Design Basis Earthquake (DBE), Maximum Considered Earthquake (MCE), Spectral Element Method, Simplified Linear Method, SAP2000 Object-based Structural Software.

\section{INTRODUCCIÓN}

Luego de los sismos de Northridge (USA, 1994), Kobe (Japón, 1995), Maule (Chile, 2010) y principalmente Tohoku (Japón, 2011), el número de construcciones con sistemas de control pasivo aumentó considerablemente en éstos países con alta peligrosidad sísmica, por el buen comportamiento que tuvieron estas construcciones al momento del cataclismo, a diferencia de algunas estructuras tradicionales que no siguieron lo prescrito en las normativas sísmicas, o en otras estructuras que a pesar de haber seguido normas de diseño sísmico tuvieron grandes pérdidas y daños en elementos estructurales y no estructurales. Saito et al. (2012), Zayas (2013), Retamales et al. (2015), Chistopupoulus, C. y Filiatraul (2006).

Dentro de los sistemas de control pasivo se tiene a los aisladores sísmicos, enfoque del presente artículo para dos alternativas de cálculo estructural que son: Estructuras Rígidas y Estructuras Flexibles; por lo tanto, según Naeim and Kelly (1999) exponen que básicamente los aisladores más comunes son de dos tipos: elastoméricos y de péndulo friccional.

Los aisladores elastoméricos más utilizados son: de bajo amortiguamiento (LDR); alto amortiguamiento (HDR) y elastoméricos con núcleo de plomo (LRB). Por otra parte, para los aisladores de triple péndulo de fricción se tiene los de simple curvatura, doble curvatura y triple curvatura que son los conocidos como FPT. Constantinou et al. (2006); Kawamura et al. (2000).

En el proyecto "Fortalecimiento de la Universidad de las Fuerzas Armadas ESPE - sede Santo Domingo", en el edificio "Vinculación con la Sociedad", se tiene un bloque estructural de 5 pisos de altura registrado como I ZE, el mismo que se decidió calcular con dos alternativas de diseño sismo resistente que son: Estructura Flexible y Estructura Rígida utilizando aisladores sísmicos FPT sobre la cimentación, para lo cual se aplicó tres métodos de diseño sísmico: Método 
Espectral, Simplificado Lineal y el software computacional SAP2000. Para el edificio evaluado en ésta investigación se planteó un aislador de gran amplitud horizontal debido al terreno dónde será cimentado siendo así un perfil de suelo tipo D.

El aislador asignado es: FPT8836/14-12/10-7, como se indica en la figura 1, con una capacidad de desplazamiento máxima de $58.89 \mathrm{~cm}$; para tener ésta traslación con uno de doble curvatura las dimensiones del aislador tendrán que ser mayores, y para el de simple curvatura mucho más. Las dimensiones respectivas del aislador FPT se presentan en la tabla 1. (Constantinou et al. 2016; Aguiar et al 2016).

Al tomar la decisión de utilizar dos modelos de cálculo, se determina como Estructura Flexible a la estructura compuesta por columnas de hormigón armado más aisladores sísmicos FPT, y como Estructura Rígida la misma que se compone por muros de corte, columnas de hormigón armado más aisladores sísmicos FPT y de ésta manera determinar cuál de los dos modelos presenta mayor factibilidad en diseño y construcción.

En la figura 2 se ilustra, la ubicación del bloque estructural I ZE del nuevo edificio "Vinculación con la Sociedad" en combinación con los demás bloques estructurales que conforman el proyecto; el mismo que estará destinado para oficinas y aulas generales que pertenecerán a la Carrera de Ingeniería Agropecuaria de la Universidad de las Fuerzas Armadas ESPE - campus Santo Domingo; localizado en la parte central del conjunto de bloques.

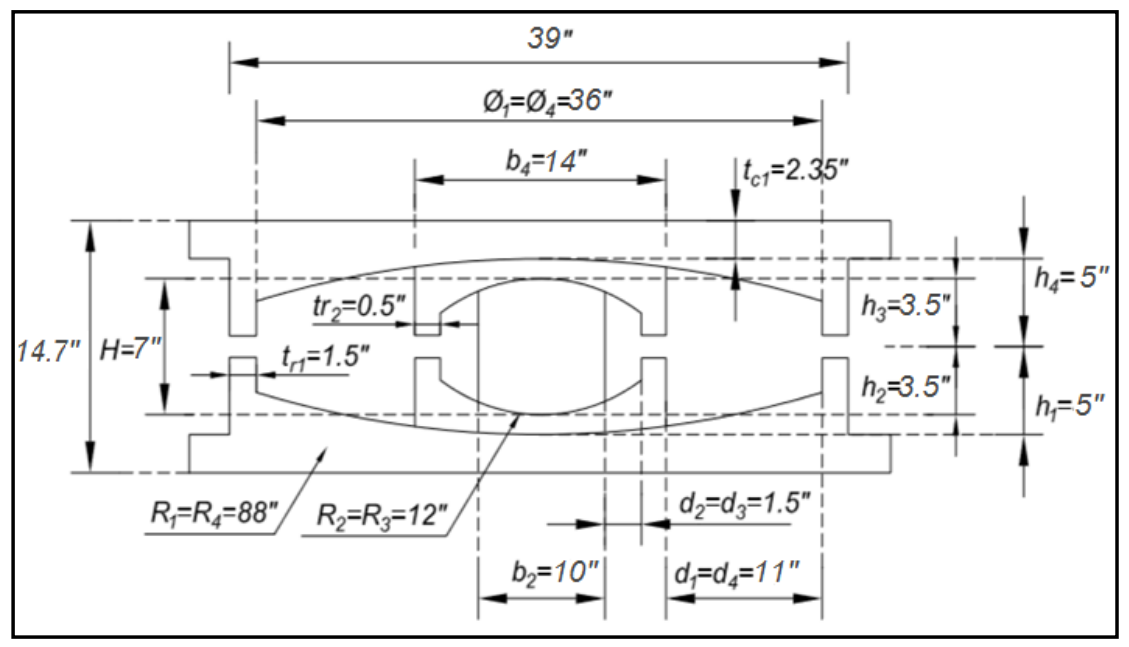

Figura 1. Geometría Aislador de Triple Péndulo de Fricción. FPT8836/14-12/10-7. Para el bloque I ZE del nuevo edificio "Vinculación con la Sociedad", UFA ESPE - campus Santo Domingo. 
Tabla 1. Detalle y dimensiones del Aislador de Triple Péndulo de Fricción FPT8836/14-12/10-7.

\begin{tabular}{|c|c|c|}
\hline Descripción & $\begin{array}{l}\text { Valor en } \\
\text { pulgadas }\end{array}$ & $\begin{array}{c}\text { Valor en } \\
\text { cm }\end{array}$ \\
\hline Radio de curvatura de las placas 1 y $4 \rightarrow \mathbf{R} \mathbf{1 = R 4}$ & $88 "$ & 223,52 \\
\hline Diámetro de las placas 1 y $4 \rightarrow \boldsymbol{\Phi 1}=\mathbf{\Phi 4}$ & $36 "$ & 91,44 \\
\hline Diámetro de aislador interior $\rightarrow$ b4 & $14 "$ & 35,56 \\
\hline Radio del aislador interior $\rightarrow \mathbf{R} \mathbf{2}=\mathbf{R} \mathbf{3}$ & $12 "$ & 30,48 \\
\hline Diámetro de deslizador rígido $\rightarrow$ b2 & $10 "$ & 25,40 \\
\hline Altura de aislador interior $\rightarrow \mathbf{H}$ & 7" & 17,78 \\
\hline Espesor de topes exteriores $\rightarrow \operatorname{tr} 1$ & $1,5 "$ & 3,81 \\
\hline Espesor de topes interiores $\rightarrow$ tr2 & $0,5 "$ & 1,27 \\
\hline Espesor de aislador exterior en el centro $\rightarrow$ tc 1 & $2,35 "$ & 5,97 \\
\hline Espesor de aislador interior en el centro $\rightarrow$ tc2 & $1,5^{\prime \prime}$ & 3,81 \\
\hline Capacidad de desplazamiento $\rightarrow \mathbf{d} \mathbf{1}=\mathbf{d} \mathbf{4}$ & $11 "$ & 27,94 \\
\hline Capacidad de desplazamiento $\rightarrow \mathbf{d} 2=\mathbf{d} 3$ & $1,5 "$ & 3,81 \\
\hline Altura de aislador exterior $\rightarrow \mathrm{h} 1=\mathrm{h} 4$ & $5 "$ & 12,70 \\
\hline Altura de aislador interior $\rightarrow$ h2= h3 & $3,5 "$ & 8,89 \\
\hline Base total del aislador $\rightarrow$ BT & $39 "$ & 99,06 \\
\hline Altura total del aislador $\rightarrow \mathbf{H T}$ & $14,7 "$ & 37,34 \\
\hline
\end{tabular}

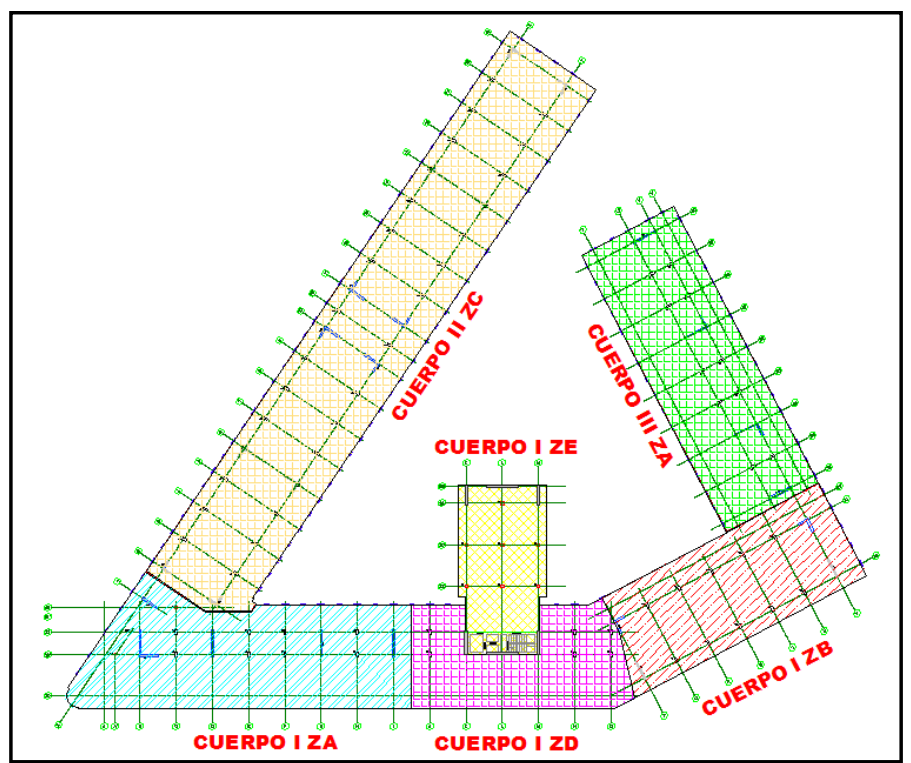

Figura 2. Ubicación del bloque estructural I ZE del nuevo edificio "Vinculación con la Sociedad" de la UFA ESPE - Santo Domingo. 


\section{MARCO TEÓRICO}

\subsection{MÉTODO MODAL - ESPECTRAL}

Primero se define el modelo constitutivo con el que se va a trabajar el aislador. Para aisladores sísmicos de triple péndulo de fricción FPT, se tienen varios modelos planteados, los que más se destacan son los propuestos por Fenz y Constantinou (2007, 2008), Fadi y Constantinou (2010).

En éste estudio se va a trabajar con el modelo de tres fases desarrollado por Mc Vitty y Constantinou (2015), no sin antes mencionar que éste modelo de cálculo es adecuado para el aislador FTP8836/14-12/10-7, indicado en la figura 1, de tal manera que de las 12 propiedades geométricas que tiene el aislador se reducen a 6 (porque algunas dimensiones son iguales) $R_{1}=R_{4}$; y $R_{2}=R_{3}$; lo mismo se tiene con las alturas $h_{1}=h_{4} ;$ y $h_{2}=h_{3}$; y con los desplazamientos $d_{1}=$ $d_{4}$; y $d_{2}=d_{3}$. Los 4 coeficientes de roce o conocidos también como coeficientes de fricción se disminuyen a 2 , el de las placas externas que se denomina a $\left(\mu_{1}=\mu_{4}\right)$ y el de las placas internas a $\left(\mu_{2}=\mu_{3}\right)$.

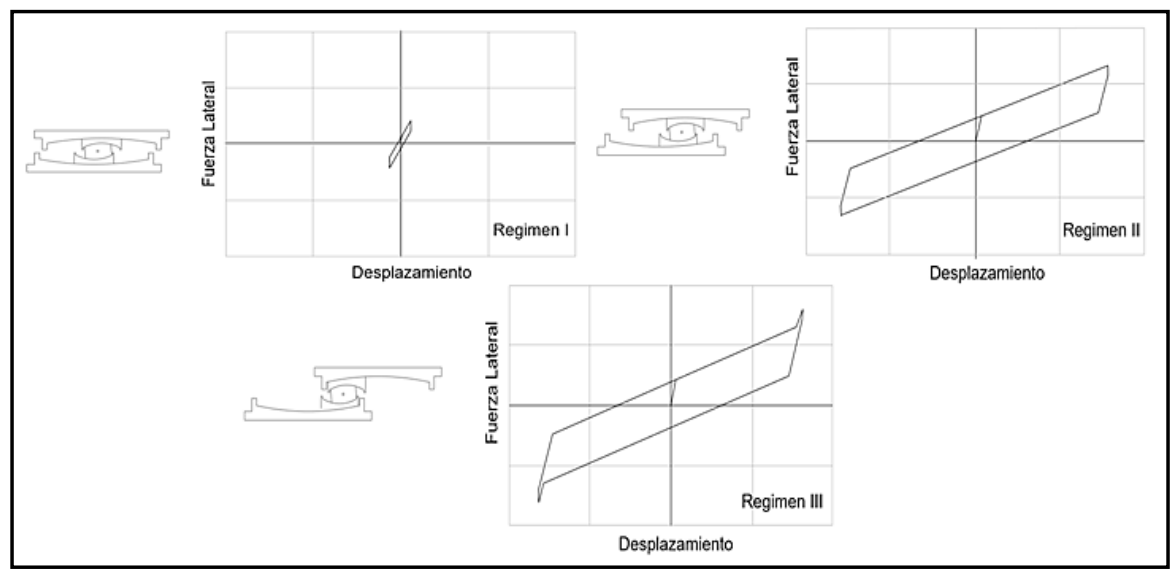

Figura 3. Desempeño del aislador en Régimen I, Régimen II y Régimen III. Fuente: Mc Vitty y Constantinou (2015).

\subsection{MÉTODO SIMPLIFICADO LINEAL}

La norma sísmica de aislación de Chile INN (2013) permite realizar un Análisis Simplificado Lineal, si se cumplen las siguientes condiciones:

i) La estructura se halla ubicada a más de $10 \mathrm{~km}$, de todas las fallas activas.

ii) La estructura está ubicada en un suelo I o II (suelo de gran resistencia a la compresión).

iii) La estructura tenga menos de 5 pisos y una altura menor a $20 \mathrm{~m}$. 
iv) El período efectivo de la estructura aislada para el sismo máximo considerado MCE sea menor o igual a $3.0 \mathrm{~s}$.

v) El período efectivo de la estructura aislada sea mayor que tres veces el período de la estructura con base fija.

vi) La superestructura tenga una configuración regular.

vii) La rigidez efectiva para el sismo de diseño $\boldsymbol{D B E}$ es mayor de un tercio de la rigidez efectiva para un $20 \%$ del desplazamiento de diseño.

viii) El sistema de aislación tiene la capacidad de producir una fuerza restitutiva tal que la sumatoria de las fuerzas laterales para el sismo de diseño sea mayor a la sumatoria de las fuerzas laterales que producen el $50 \%$ del desplazamiento lateral de diseño más $0.025 \mathrm{~W}$. Siendo $\mathrm{W}$ el peso total de la carga vertical completa.

ix) La curva de histéresis del sistema de aislación son independientes de la velocidad de carga; el sistema de aislación tiene propiedades tal que la curva de histéresis no depende de la magnitud de la carga vertical y efectos de solicitaciones bidireccionales.

x) El sistema de aislación debe tener una capacidad de desplazamiento mayor a la que se espera con el sismo máximo considerado y mayor a 1.2 veces mayor que el desplazamiento total de diseño.

Por otra parte, la norma INN (2013) permite realizar un análisis espectral si la estructura se encuentra ubicada en un perfil de suelo I, II, y III. Además cumple con las condiciones indicadas en el párrafo anterior.

\section{- Descripción y análisis del Método Simplificado Lineal}

Se considera, en forma aproximada que una estructura se desplaza como cuerpo rígido al encontrarse sobre aisladores sísmicos y ante el movimiento del suelo en sentido " $x$ ", ver figura 4 a); por lo tanto, se considera el nuevo sistema con un grado de libertad para un modelo constitutivo del aislador bilineal como se indica en la figura 4 b).

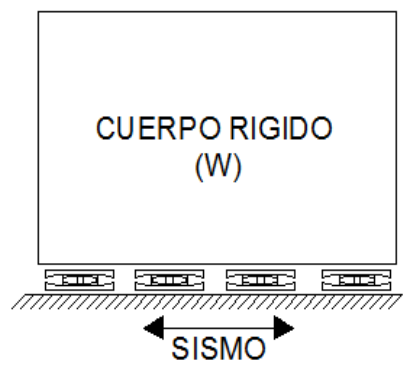

a)

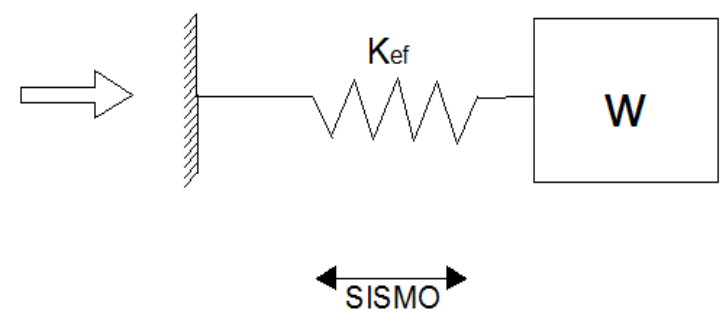

b)

Figura 4. a) Edificio sobre aisladores sísmicos; b) Modelo de un grado de libertad. 
El sistema de un grado de libertad, está definido por una rigidez efectiva $K_{e f}$, factor de amortiguamiento $\xi_{\text {ef }}$ y el peso total que gravita sobre el sistema de aislación $W$. Se considera que la relación constitutiva entre la fuerza y el desplazamiento es bilineal, ver figura 5 .

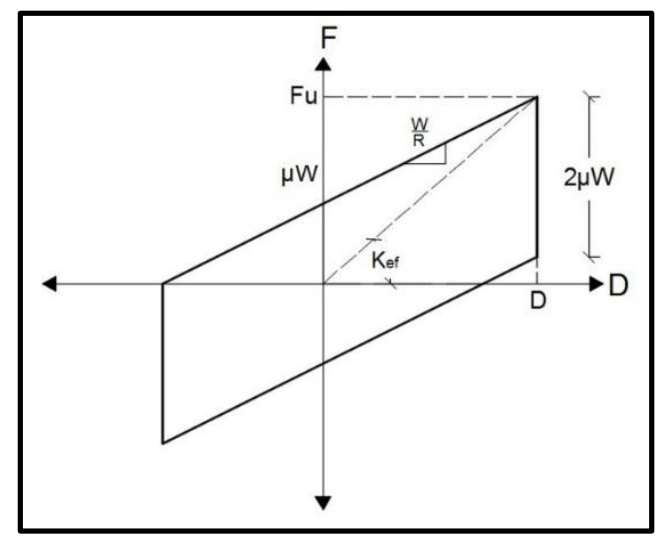

Figura 5. Modelo constitutivo bilineal para un aislador FPT.

Estrictamente la relación constitutiva indicada en la figura 5, es para un aislador de péndulo de fricción de primera generación o de simple curvatura, pero como aproximación se la utiliza para el de triple péndulo de fricción. En éste modelo se aprecia que, la resistencia característica vale $\mu W$, donde $\mu$ es el coeficiente de roce y la rigidez tangente es igual a $k_{p}=W / R$. Siendo $R$ el radio de curvatura del aislador. La fuerza $F_{u}$ asociada al desplazamiento $D$, vale:

$$
F_{u}=\mu W+\frac{W}{R} D
$$

La rigidez efectiva $k_{e f}$, se determina mediante la relación entre la fuerza $F_{u}$ y el desplazamiento $D$ así: (ASCE 7-10)

$$
k_{e f}=\frac{W}{R}+\frac{\mu W}{D}
$$

Por otra parte, el factor de amortiguamiento $\xi_{\text {ef }}$ se encuentra dividiendo la energía disipada en un lazo del diagrama de histéresis $E_{D}$ para $4 \pi E_{S}$; siendo $E_{S}$ la energía elástica.

$$
\xi_{e f}=\frac{E_{D}}{4 \pi E_{S}}=\frac{(2 \mu D)(2 D)}{4 \pi \frac{F_{u} D}{2}}
$$

De donde se halla:

$$
\xi_{\text {ef }}=\frac{2}{\pi}\left[\frac{\mu}{\mu+\frac{D}{R}}\right]
$$


Por otra parte, el período T y el desplazamiento $D$ del sistema de un grado de libertad se encuentran con las siguientes ecuaciones:

$$
\begin{aligned}
& T=2 \pi \sqrt{\frac{W}{k_{e f} g}} \\
& D=\left(\frac{S_{1} * g}{4 \pi^{2}}\right) \frac{T}{B}
\end{aligned}
$$

Las variables no definidas aún, son $g$ que es la aceleración de la gravedad; $S_{1}$ es el coeficiente de la aceleración de la gravedad encontrada en el espectro de diseño elástico DBE según el perfil de suelo del lugar, para un período de 1 segundo; el bloque estructural I ZE se encuentra en Santo Domingo, donde el factor de peligrosidad sísmica $z=0.4$, sobre un perfil de suelo clasificado como $D$, y en consecuencia los factores de sitio de aceleración, desplazamiento y de comportamiento no lineal del suelo son: $F_{a}=1.2 ; F_{d}=1.19 ; F_{s}=1.28$. Y finalmente, $B$ es el factor que permite obtener espectros para cualquier valor de amortiguamiento, los valores recomendados por el ASCE 7-10 se indican a continuación en la tabla 2.

Tabla 2. Valores de reducción del factor $B$ que permite encontrar espectros con amortiguamientos diferentes al 5\%. Fuente: ASCE 7-10.

\begin{tabular}{|c|c|c|c|c|c|c|c|}
\hline $\begin{array}{c}\xi_{\text {ef }} \\
(\%)\end{array}$ & $\leq 2$ & 5 & 10 & 20 & 30 & 40 & $\geq 50$ \\
\hline $\boldsymbol{B}$ & 0.8 & 1.0 & 1.2 & 1.5 & 1.7 & 1.9 & 2.0 \\
\hline
\end{tabular}

El cálculo del desplazamiento $D$ se realiza en forma iteractiva, para el efecto se impone un desplazamiento inicial; con este valor se halla la rigidez efectiva y amortiguamiento equivalente, mediante las ecuaciones (2) y (3). Posteriormente se encuentra el valor de $B$, en la tabla 2 , con el que se encuentra el espectro asociado al amortiguamiento $\xi_{e f}$. Luego se determina el período $T$ y se recalcula el desplazamiento, con las ecuaciones (4) y (5).

Se comparan los desplazamientos (el impuesto y el recalculado) y si son menor a una tolerancia se habrá encontrado el desplazamiento $D$, la rigidez efectiva $k_{e f}$, el período $T$, y el amortiguamiento equivalente $\xi_{e f}$. Para una convergencia más rápida se aplica el Método de la Bisección.

Sea $D_{D}, D_{M}$, los desplazamientos laterales del sistema de aislación ante los sismos de diseño $D B E$ y máximo considerado $M C E$; en la forma presentada se lo realizó en forma general, por este motivo en la ecuación (5) se escribió $S_{1}$. Para cuando se trabaja con el sismo DBE se tiene $S_{D 1}$ y para el sismo MCE se tiene 
$S_{M 1}$, que son las ordenadas espectrales para un período de 1 segundo halladas con los espectros DBE y $M C E$.

Ahora bien, el desplazamiento de diseño total $D_{T D}$, y el desplazamiento máximo total $D_{T M}$ debe incorporar los desplazamientos adicionales por torsión natural y accidental, que se encuentra, con la siguiente ecuación para el sismo DBE y para el sismo MCE es algo similar.

$$
D_{T D}=D_{D}\left[1+y \frac{12 e}{b^{2}+d^{2}}\right]
$$

Donde: $b, d$ son las dimensiones en planta de la estructura; $e$ es la excentricidad medida en planta entre el centro de masas de la superestructura y el centro de rigidez del sistema de aislación más la excentricidad accidental igual al $5 \%$ de la dimensión máxima, de la losa de aislación, perpendicular a la dirección del análisis sísmico. (INN, 2013); y es la distancia entre el centro de rigidez del sistema de aislación y el punto de interés, medida perpendicular a la dirección del análisis sísmico. Constantinou (2015) recomienda que el factor de amplificación del desplazamiento se considere entre 1.1 y 1.2 .

El desplazamiento mínimo del sistema de aislación será mayor al desplazamiento que se encuentra en el espectro de desplazamiento, para el período de la estructura aislada dividida para $B$.

De la figura 4 b), se deduce que el cortante en el sistema de aislación, es igual a la sumatoria de las rigideces efectivas $k_{e f}$ de los aisladores por el desplazamiento $D$, esto sería para el sistema de aislación.

$$
V_{b}=\frac{\sum k_{e f} \max D_{D}}{R_{D}}
$$

Donde $V_{b}$ es el cortante basal en el sistema de aislación; $k_{e f} \max$ es la rigidez efectiva hallada con los máximos valores de coeficiente de roce; $R_{D}$ será mayor a 1.5 para los elementos de la fundación y 1 para el sistema de aislación. ecuación.

El cortante mínimo para la superestructura $V_{s}$ se halla con la siguiente

$$
V_{s}=\frac{\sum k_{e f} \max D_{D}}{R_{S}}
$$

La variable todavía no definida de la ecuación (8), es el factor de reducción de respuesta por la sobre resistencia que tiene la estructura $R_{S}$, que la norma INN (2013) recomienda igual a 2 para estructuras de acero y/o hormigón con altura menor a $35 \mathrm{~m}$. Para estructuras en voladizo $R=1.4$, si la altura es menor a $10 \mathrm{~m}$. 
Finalmente las fuerzas en cada uno de los pisos, se halla con la siguiente expresión. (ASCE 7-10).

$$
F_{x}=V_{s} \frac{W_{x} h_{x}}{\sum_{i}^{n} W_{i} h_{i}}
$$

Donde: $F_{x}, W_{x}, h_{x}$ son la fuerza horizontal, el peso y la altura desde la base al piso $x ; W_{i}$ es el peso del piso $i ; h_{i}$ es la altura desde el nivel del suelo hasta el piso $i$.

Otra alternativa de cálculo, en forma directa para las fuerzas de cada uno de los pisos, es la relación entre el cortante basal en el sistema de aislación $V_{b}$ y el número de pisos correspondiente a la estructura $N P$ obteniendo como resultado las fuerzas laterales iguales en cada piso.

$$
F_{x}=\frac{V_{b}}{N P}
$$

\subsection{USO DE SAP2000}

Hasta hace poco no se contaba con modelos aplicables de histéresis, o elementos lineales y no lineales que estén disponibles en el software estructural SAP2000 que puedan simular exactamente al modelo del aislador de Triple Péndulo de Fricción FPT, por lo tanto se propusieron varios modelos de aisladores para representar su comportamiento; sin embargo, la conducta del aislador de Triple Péndulo de Fricción no es exactamente como la exponen debido a su forma cóncava de péndulo de fricción pero si es muy parecida. Por consiguiente CSi ya ha publicado en las últimas versiones del software la incorporación de la opción directa para modelar aisladores de triple péndulo. (Khloud El-Bayoumi, 2015).

Se ilustra el modelamiento del aislador de Triple Péndulo de Fricción (FPT) en el programa comercial de cálculo de estructuras SAP2000 para los dos modelos expuestos que son: Estructura Flexible y Estructura Rígida, tomando en consideración todos los datos utilizados para un Análisis Lineal como se lo dedujo con el Método de Superposición Modal, el mismo que fue calculado empleando la librería computacional CEINCI-LAB mediante el software matemático MATLAB. Es decir se presenta la descripción de cómo introducir los parámetros de entrada del aislador FPT cómo rodamiento para cada estructura modelada. Unidades de medida utilizadas en SAP2000 [Tonf, m].

Cabe recalcar que el mismo proceso se lo realiza tanto en la Estructura Flexible como para la Rígida únicamente con diferentes datos e incluso para cada tipo de estructura, se debe cargar al programa SAP2000 el espectro reducido para $B$ correspondiente a cada evaluación sísmica DBE y MCE para cada modelo; por 
lo tanto para la zona de períodos largos mayores a 2 segundos, el espectro elástico se divide para el valor de $B$, el mismo que es función del factor de amortiguamiento de los aisladores. Constantinou et al. (2011).

$$
B=\left(\frac{\xi_{e q}}{0.05}\right)^{0.3}
$$

\section{- $\quad$ Modelo Estructural (BLOQUE I ZE)}

Edificio de 6 pisos de altura incluido losa de aislación, conformado por secciones de columnas y vigas de hormigón armado iguales para los dos modelos: (80x80) cm columnas para el 1ero y 2 do piso, $(70 \times 70) \mathrm{cm}$ columnas para el resto de pisos y vigas de $(40 \times 50) \mathrm{cm}$; adicionalmente para la Estructura Rígida se colocó muros de corte de $30 \mathrm{~cm}$ de espesor.

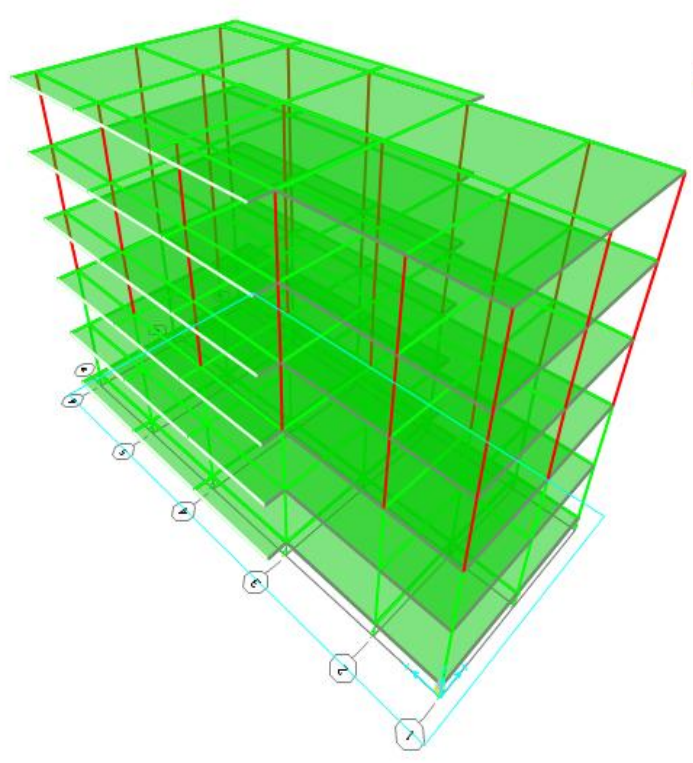

a) Estructura Flexible

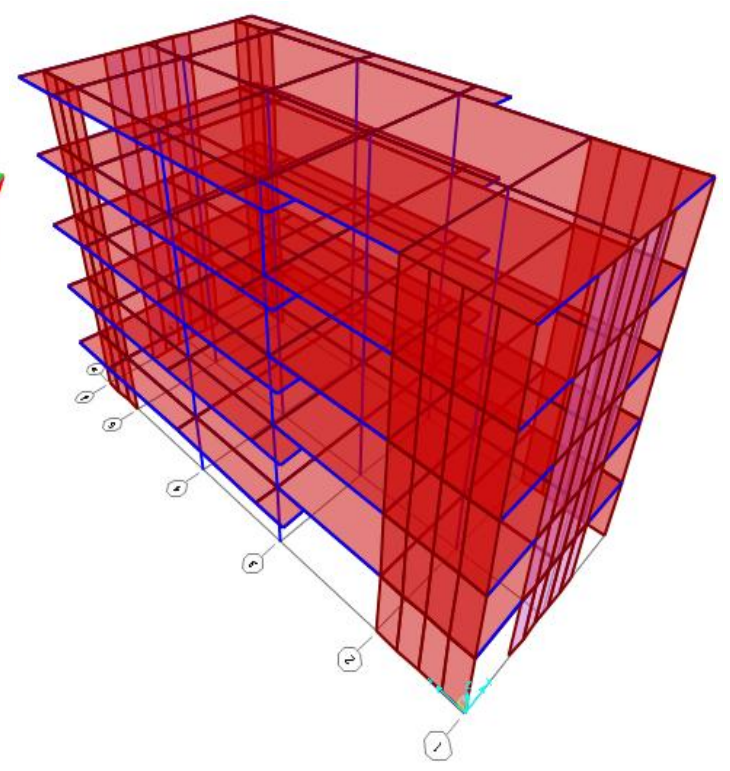

b) Estructura Rígida

Figura 6. Vista en 3D Estructura Flexible y Estructura Rígida - Modelo SAP2000.

Para determinar los parámetros de ingreso para cada aislador FPT, se necesita la carga vertical que llega a cada uno; para lo cual se recomienda realizar una tabla con cada peso y rigidez efectiva; rigidez que fue calculada con el modelo de tres fases de Mc Vitty y Constantinou (2015) que la vez se obtuvo el factor de amortiguamiento y el desplazamiento máximo para cada condición, según corresponda al sismo de análisis DBE y MCE. 


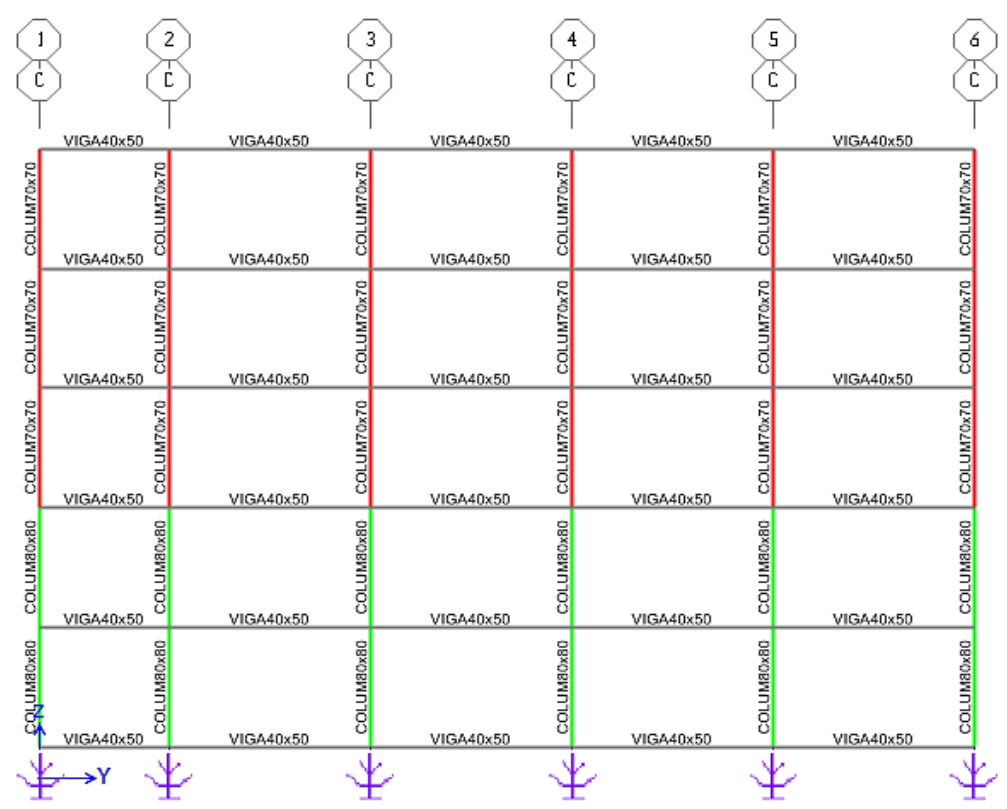

Figura 7. Vista en 2D (dirección y-z) Estructura Flexible - Modelo SAP2000.

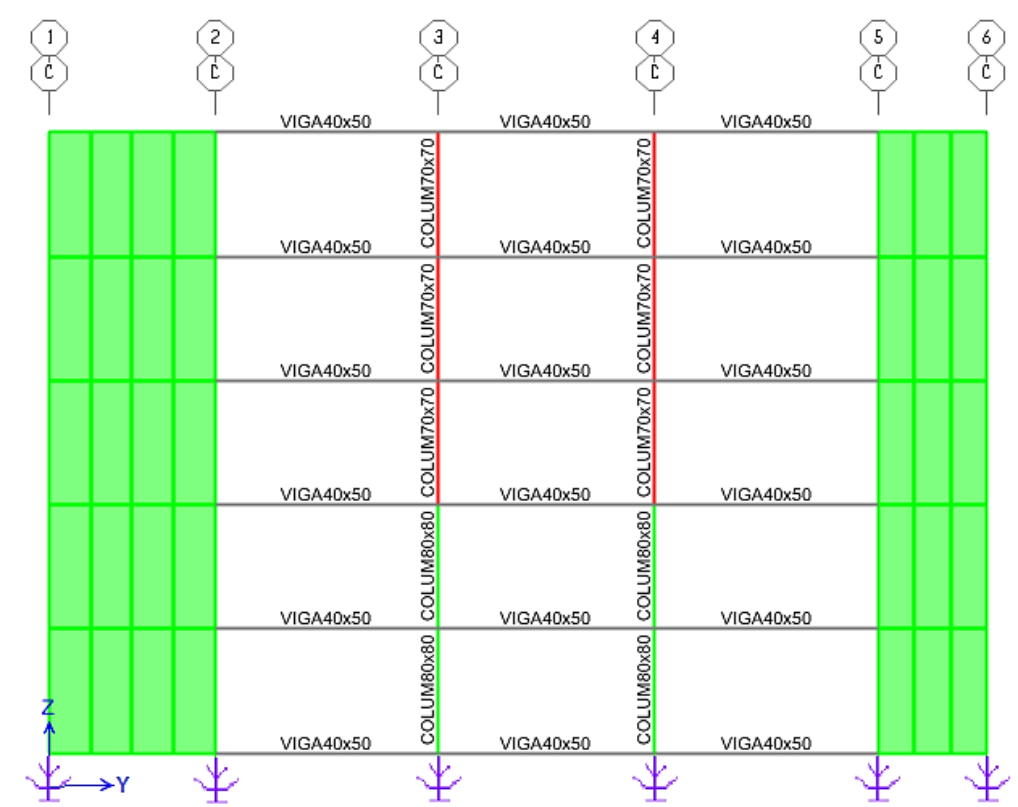

Figura 8. Vista en 2D (dirección y-z) Estructura Rígida - Modelo SAP2000.

\section{- FPT aislador}

Se recomienda preparar y calcular en una hoja de excel las propiedades del aislador, fórmulas y cálculos respectivos que necesita el SAP2000 para modelar un aislador FPT como lo indica Khloud El-Bayoumi (2015) a continuación: 
Tabla 3. Propiedades geométricas del aislador FPT8836/14-12/10-7

(Ver figura 1 y tabla 1 ).

\begin{tabular}{|c|c|c|c|c|}
\hline \multicolumn{5}{|c|}{ Propiedades Geométricas del aislador FPT } \\
\hline R1=R4= & 2235.20 & $\mathrm{~mm}$ & 7.3333 & $\mathrm{ft}$ \\
\hline$R 2=R 3=$ & 304.80 & $\mathrm{~mm}$ & 1.0000 & $\mathrm{ft}$ \\
\hline h1=h4= & 127.00 & $\mathrm{~mm}$ & 0.4267 & $\mathrm{ft}$ \\
\hline h2=h3= & 88.90 & $\mathrm{~mm}$ & 0.2917 & $\mathrm{ft}$ \\
\hline$d 1=d 4=$ & 279.40 & $\mathrm{~mm}$ & 0.9167 & $\mathrm{ft}$ \\
\hline $\mathrm{d} 2=\mathrm{d} 3=$ & 38.10 & $\mathrm{~mm}$ & 0.1250 & $\mathrm{ft}$ \\
\hline b2= & 254.00 & $\mathrm{~mm}$ & 0.8333 & $\mathrm{ft}$ \\
\hline b4= & 355.60 & $\mathrm{~mm}$ & 1.1667 & $\mathrm{ft}$ \\
\hline htotal= & 373.40 & $\mathrm{~mm}$ & 1.2251 & $\mathrm{ft}$ \\
\hline
\end{tabular}

\section{Radio Efectivo}

$$
\begin{aligned}
& R_{1 e f f}=R_{4 e f f}=R_{1}-h_{1}=2108.2 \mathrm{~mm}=6.9167 \mathrm{ft} \\
& R_{2 e f f}=R_{3 e f f}=R_{2}-h_{2}=215.90 \mathrm{~mm}=0.7083 \mathrm{ft}
\end{aligned}
$$

\section{Capacidad de desplazamiento real}

$d_{1}{ }^{*}=d_{4}{ }^{*}=d_{1} * \frac{R_{1 e f f}}{R_{1}}=263.525 \mathrm{~mm}=0.8646 \mathrm{ft}$

$d_{2}{ }^{*}=d_{3}{ }^{*}=d_{3} * \frac{R_{2 e f f}}{R_{2}}=26.9875 \mathrm{~mm}=0.0885 \mathrm{ft}$

Módulo de Elasticidad $\rightarrow E=21000000 \mathrm{~T} / \mathrm{m}^{2}=20594.07 \mathrm{KN} / \mathrm{cm}^{2}$

\section{Propiedades friccionales del aislador}

Datos obtenidos a partir del fabricante o por fórmulas equivalentes; en la presente investigación el análisis se lo realizó con los coeficientes de roce máximos encontrados experimentalmente, por lo que más adelante se presenta los coeficientes de roce establecidos. Ver tabla 9.

$\mu_{1}=\mu_{4}$ Má $x=0.0685 \rightarrow$ Límite superior

$\mu_{1}=\mu_{4}$ Mín $=0.0547 \rightarrow$ Límite inferior

$\mu_{2}=\mu_{3}$ Máx $=0.0157 \rightarrow$ Límite superior

$\mu_{2}=\mu_{3}$ Mín $=0.0099 \rightarrow$ Límite inferior

\section{Cálculo desplazamiento máximo}

Para calcular el desplazamiento máximo, se aplican las fórmulas descritas en el apartado 2.2 del Método Simplificado Lineal del ASCE 7-10, dónde describe el proceso interactivo para determinar un desplazamiento recalculado equivalente, por lo tanto para la presente investigación se lo realizó mediante una rutina en MATLAB, obteniendo así: desplazamiento $D$, rigidez efectiva $k_{e f}$, período $T$, y el factor de amortiguamiento equivalente $\xi_{\text {ef }}$ para cada aislador. Ver tabla 4. 
Tabla 4. Valores constantes de periodo, amortiguamiento y desplazamiento máximo para sismo DBE y MCE de todos los aisladores para las dos estructuras.

\begin{tabular}{|c|c|c|}
\hline \multirow{2}{*}{$\begin{array}{l}\text { Propiedades } \\
\text { aislador }\end{array}$} & \multirow{2}{*}{$\frac{\text { SISMO DBE }}{\text { SD1 }=0.6032}$} & SISMO MCE \\
\hline & & SD1 $=1.5 * 0.6032$ \\
\hline Desplaza. q & $24.8044 \mathrm{cn}$ & $47.4137 \mathrm{~cm}$ \\
\hline Amortig. $\beta$ & 0.3067 & 0.2206 \\
\hline Periodo T & 2.8614 & 3.2971 \\
\hline
\end{tabular}

\section{Inercia Rotacional 1 del aislador}

Se calcula la inercia rotacional a partir de la rigidez efectiva de cada aislador y $h$ corresponde a la altura total del aislador. Ver tabla 3.

$$
I=\frac{K_{e f f} * h^{3}}{12 E}
$$

\section{Masa del aislador}

Para determinar la masa del aislador se necesita el desplazamiento máximo $D D^{\prime}{ }_{\text {máx }}$ encontrado (ver tabla 4). Según lo señala Khloud El-Bayoumi (2015) se aplica una correlación tamaño/peso del aislador considerado como un cilindro dónde $D$ en $(f t)$, para el efecto se aplica las siguientes ecuaciones:

$$
\begin{array}{cr}
D=2 * D D_{\text {máx }}^{\prime} & \\
W=0.241 D^{2}-0.0564 D & {[\mathrm{klb}]} \\
\therefore \quad & M=\frac{W[\text { Ton }]}{g} \quad\left[\text { Ton. } \frac{\mathrm{seg}^{2}}{\mathrm{~m}}\right]
\end{array}
$$

A partir de obtener todos estos datos, es importante mencionar que se aplica al programa SAP2000 un Análisis Lineal el mismo que fue resuelto con el Método de Superposición Modal utilizando CEINCI-LAB. Cabe resaltar que todo el cálculo de datos en los aisladores para su ingreso al SAP2000, se consideró las unidades de medida según como lo indica Khloud El-Bayoumi (2015), teniendo en cuenta que todo el modelamiento para cada estructura se trabajó con: [Tonf, m] como unidad de medida en el programa comercial. Ver tablas $5,6,7$ y 8.

Al utilizar el programa SAP2000, únicamente se trabajó con las propiedades en dirección U2 y U3 de las opciones desplegables por el software para un aislador de triple péndulo por ser un análisis lineal; por lo tanto los datos del aislador que se ingresan al SAP2000 son: Inercia Rotacional 1, Masa, Rigidez Efectiva y Amortiguamiento; se indica un resumen total en las tablas 5, 6, 7 y 8 para cada estructura modelada con el sismo correspondiente DBE y MCE. 
Tabla 5. Datos para ingresar a SAP2000 - Aisladores para Estructura Flexible con el sismo DBE.

\begin{tabular}{|c|c|c|c|c|c|}
\hline \multirow{2}{*}{$\begin{array}{l}\text { Aislador } \\
\text { Tipo }\end{array}$} & \multirow{2}{*}{$\begin{array}{c}\text { W } \\
(\text { Ton })\end{array}$} & \multirow{2}{*}{$\begin{array}{c}\text { Inercia } \\
\text { Rotacional } 1 \\
\left(\mathrm{~m}^{\mathbf{4}}\right)\end{array}$} & \multirow{2}{*}{$\begin{array}{c}\text { Masa } \\
\left.\text { Ton. } \frac{\text { seg }^{2}}{m}\right)\end{array}$} & \multicolumn{2}{|c|}{$\begin{array}{c}\text { Propiedades para dirección } \\
\text { U2 y U3 (Horizontal) }\end{array}$} \\
\hline & & & & keff & Amortig. $\beta$ \\
\hline 1 & 28.1794 & 2.8614E-09 & 0.0253 & 13.8500 & 0.3067 \\
\hline 2 & 69.7069 & 7.0801E-09 & 0.0253 & 34.2700 & 0.3067 \\
\hline 3 & 97.8863 & 9.9435E-09 & 0.0253 & 48.1300 & 0.3067 \\
\hline 4 & 110.7400 & 1.1249E-08 & 0.0253 & 54.4500 & 0.3067 \\
\hline 5 & 59.3250 & 6.0264E-09 & 0.0253 & 29.1700 & 0.3067 \\
\hline 6 & 52.6015 & 5.3426E-09 & 0.0253 & 25.8600 & 0.3067 \\
\hline 7 & 130.1195 & $1.3218 \mathrm{E}-08$ & 0.0253 & 63.9800 & 0.3067 \\
\hline 8 & 155.0360 & 1.5749E-08 & 0.0253 & 76.2300 & 0.3067 \\
\hline 9 & 83.0550 & 8.4374E-09 & 0.0253 & 40.8400 & 0.3067 \\
\hline
\end{tabular}

Tabla 6. Datos para ingresar a SAP2000 - Aisladores para Estructura Flexible con el sismo MCE.

\begin{tabular}{|c|c|c|c|c|c|}
\hline \multirow{2}{*}{$\begin{array}{l}\text { Aislador } \\
\text { Tipo }\end{array}$} & \multirow{2}{*}{$\begin{array}{c}\text { W } \\
(\text { Ton })\end{array}$} & \multirow{2}{*}{$\begin{array}{c}\text { Inercia } \\
\text { Rotacional } 1 \\
\left(m^{4}\right)\end{array}$} & \multirow{2}{*}{$\begin{array}{c}\text { Masa } \\
\left(\begin{array}{c}\text { Ton. } \frac{\text { seg }^{2}}{m}\end{array}\right)\end{array}$} & \multicolumn{2}{|c|}{$\begin{array}{l}\text { Propiedades para dirección } \\
\text { U2 y U3 (Horizontal) }\end{array}$} \\
\hline & & & & keff & Amortig. $\beta$. \\
\hline 1 & 28.1794 & $2.1569 \mathrm{E}-09$ & 0.0997 & 10.4400 & 0.2206 \\
\hline 2 & 69.7069 & 5.3322E-09 & 0.0997 & 25.8100 & 0.2206 \\
\hline 3 & 97.8863 & 7.4891E-09 & 0.0997 & 36.2500 & 0.2206 \\
\hline 4 & 110.7400 & 8.4725E-09 & 0.0997 & 41.0100 & 0.2206 \\
\hline 5 & 59.3250 & 4.5389E-09 & 0.0997 & 21.9700 & 0.2206 \\
\hline 6 & 52.6015 & 4.0245E-09 & 0.0997 & 19.4800 & 0.2206 \\
\hline 7 & 130.1195 & 9.9538E-09 & 0.0997 & 48.1800 & 0.2206 \\
\hline 8 & 155.0360 & $1.1861 \mathrm{E}-08$ & 0.0997 & 57.4100 & 0.2206 \\
\hline 9 & 83.0550 & 6.3549E-09 & 0.0997 & 30.7600 & 0.2206 \\
\hline
\end{tabular}

Tabla 7. Datos para ingresar a SAP2000 - Aisladores para Estructura Rígida con el sismo DBE.

\begin{tabular}{|c|c|c|c|c|c|}
\hline \multirow{2}{*}{$\begin{array}{c}\text { Aislador } \\
\text { Tipo }\end{array}$} & $\begin{array}{c}\text { W } \\
(\text { Ton })\end{array}$ & $\begin{array}{c}\text { Inercia } \\
\text { Rotacional 1 } \\
\left(\boldsymbol{m}^{\mathbf{4}}\right)\end{array}$ & \multirow{2}{*}{$\begin{array}{c}\text { Masa } \\
\left.\text { Ton. } \frac{\boldsymbol{s e g}^{\mathbf{2}}}{\boldsymbol{m}}\right)\end{array}$} & \multicolumn{2}{|c|}{$\begin{array}{c}\text { Propiedades para dirección } \\
\text { U2 y U3 (horizontales) }\end{array}$} \\
\cline { 5 - 6 } & & keff & Amortig. $\boldsymbol{\beta}$. \\
\hline $\mathbf{1}$ & 35.5950 & $3.6154 \mathrm{E}-09$ & 0.0253 & 17.5000 & 0.3067 \\
\hline $\mathbf{2}$ & 81.5719 & $8.2866 \mathrm{E}-09$ & 0.0253 & 40.1100 & 0.3067 \\
\hline $\mathbf{3}$ & 114.2006 & $1.1600 \mathrm{E}-08$ & 0.0253 & 56.1500 & 0.3067 \\
\hline $\mathbf{4}$ & 120.6275 & $1.2253 \mathrm{E}-08$ & 0.0253 & 59.3100 & 0.3067 \\
\hline $\mathbf{5}$ & 92.9425 & $9.4414 \mathrm{E}-09$ & 0.0253 & 45.7000 & 0.3067 \\
\hline $\mathbf{6}$ & 31.6400 & $3.2146 \mathrm{E}-09$ & 0.0253 & 15.5600 & 0.3067 \\
\hline $\mathbf{7}$ & 66.4440 & $3.3747 \mathrm{E}-09$ & 0.0253 & 16.3400 & 0.3067 \\
\hline $\mathbf{8}$ & 152.2675 & $1.5466 \mathrm{E}-08$ & 0.0253 & 74.8600 & 0.3067 \\
\hline $\mathbf{9}$ & 168.8785 & $1.7154 \mathrm{E}-08$ & 0.0253 & 83.0300 & 0.3067 \\
\hline $\mathbf{1 0}$ & 130.1195 & $1.3218 \mathrm{E}-08$ & 0.0253 & 63.9800 & 0.3067 \\
\hline $\mathbf{1 1}$ & 44.2960 & $2.2498 \mathrm{E}-09$ & 0.0253 & 10.8900 & 0.3067 \\
\hline
\end{tabular}


Tabla 8. Datos para ingresar a SAP2000 - Aisladores para Estructura Rígida con el sismo MCE.

\begin{tabular}{|c|c|c|c|c|c|}
\hline \multirow{2}{*}{$\begin{array}{c}\text { Aislador } \\
\text { Tipo }\end{array}$} & $\begin{array}{c}\text { W } \\
(\text { Ton })\end{array}$ & $\begin{array}{c}\text { Inercia } \\
\text { Rotacional 1 } \\
\left(\boldsymbol{m}^{\mathbf{4}}\right)\end{array}$ & $\begin{array}{c}\text { Masa } \\
\left(\boldsymbol{T o n} \cdot \frac{\boldsymbol{s e g}^{\mathbf{2}}}{\boldsymbol{m}}\right)\end{array}$ & \multicolumn{2}{|c|}{$\begin{array}{c}\text { Propiedades para dirección } \\
\text { U2 y U3 (horizontales) }\end{array}$} \\
\cline { 5 - 6 } & & keff & Amortig. $\boldsymbol{\beta}$. \\
\hline $\mathbf{1}$ & 35.5950 & $2.7229 \mathrm{E}-09$ & 0.0997 & 13.1800 & 0.2206 \\
\hline $\mathbf{2}$ & 81.5719 & $6.2413 \mathrm{E}-09$ & 0.0997 & 30.2100 & 0.2206 \\
\hline $\mathbf{3}$ & 114.2006 & $8.7370 \mathrm{E}-09$ & 0.0997 & 42.2900 & 0.2206 \\
\hline $\mathbf{4}$ & 120.6275 & $9.2287 \mathrm{E}-09$ & 0.0997 & 44.6700 & 0.2206 \\
\hline $\mathbf{5}$ & 92.9425 & $7.1110 \mathrm{E}-09$ & 0.0997 & 34.4200 & 0.2206 \\
\hline $\mathbf{6}$ & 31.6400 & $2.4213 \mathrm{E}-09$ & 0.0997 & 11.7200 & 0.2206 \\
\hline $\mathbf{7}$ & 66.4440 & $2.5411 \mathrm{E}-09$ & 0.0997 & 12.3000 & 0.2206 \\
\hline $\mathbf{8}$ & 152.2675 & $1.1650 \mathrm{E}-08$ & 0.0997 & 56.3900 & 0.2206 \\
\hline $\mathbf{9}$ & 168.8785 & $1.2921 \mathrm{E}-08$ & 0.0997 & 62.5400 & 0.2206 \\
\hline $\mathbf{1 0}$ & 130.1195 & $9.9538 \mathrm{E}-09$ & 0.0997 & 48.1800 & 0.2206 \\
\hline $\mathbf{1 1}$ & 44.2960 & $1.6941 \mathrm{E}-09$ & 0.0997 & 8.2000 & 0.2206 \\
\hline
\end{tabular}

Los datos de inicio para ingresar al SAP2000 son: la Masa y la Inercia Rotacional 1 correspondientes para cada aislador (ver tablas 5, 6, 7 y 8), como se muestra en la figura 9, al momento de elegir Link/Support Type en: Triple Pendulum isolator, se despliegan las propiedades lineales mencionadas.

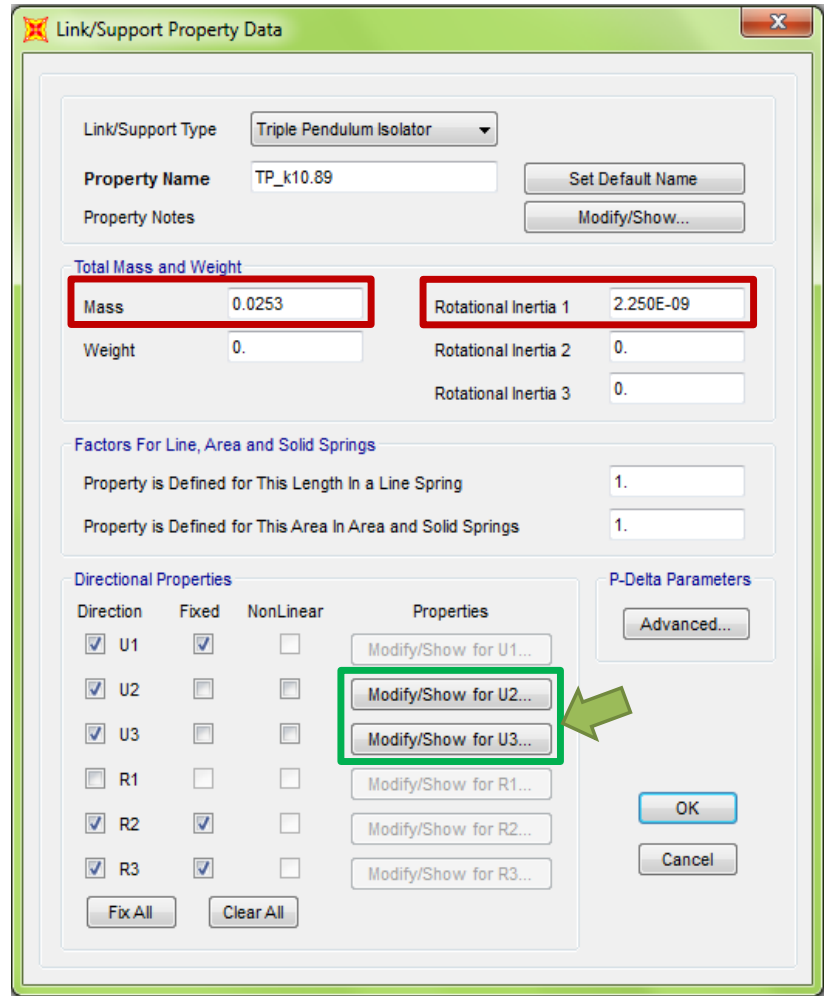

Figura 9. Link Main Properties - SAP2000. 
Y para terminar con el Análisis Lineal, se ingresa la Rigidez Efectiva y el Amortiguamiento de cada aislador, que son las propiedades para la dirección U2 y U3 (Horizontal) como se muestra en las tablas 5, 6, 7 y 8, al ingresar a la opción Modify/Show for U2 and Modify/Show for U3 como se ilustra en la figura 10.
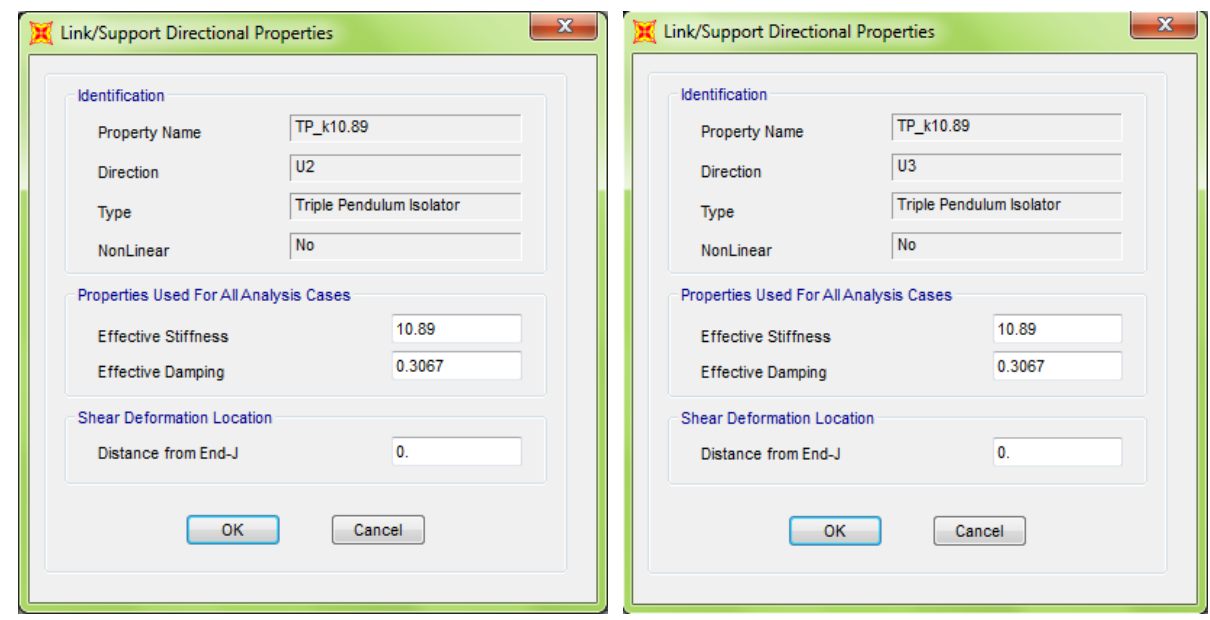

Figura 10. Link Properties in direction U2 and U3 - SAP2000.

\section{CARGAS Y COEFICIENTES DE ROCE}

La carga permanente $D$ para los pisos interiores es $D=0.80 \frac{T}{\mathrm{~m}^{2}}=$ $0.0007845 \frac{K N}{\mathrm{Cm}^{2}}$, para la cubierta $D=0.60 \frac{T}{\mathrm{~m}^{2}}=0.0005884 \frac{\mathrm{KN}}{\mathrm{Cm}^{2}}$ y la carga viva es $L=0.25 \frac{T}{\mathrm{~m}^{2}}=0.0002452 \frac{\mathrm{KN}}{\mathrm{Cm}^{2}}$. Para el análisis sísmico se trabajó con la combinación $D+0.25 \mathrm{~L}$. En la figura 11 se presenta el mosaico de cargas con el que se halló la carga vertical que llega a cada aislador, la misma que determina rigideces y factores de amortiguamiento correspondientes al modelo de 3 fases para las dos alternativas de cálculo expuestas como estructuras aisladas con y sin muros de corte.

Un dato importante para el cálculo sísmico, es indicar cuál es el coeficiente de roce con el que trabaja el aislador FPT, los mismos que fueron determinados mediante ensayos en forma experimental con valores máximos, nominales y mínimos como se detalla en la tabla 9; también se indica cual es el valor para el coeficiente de aceleración de la gravedad $S_{1}$, para el bloque I ZE correspondiente a los sismos DBE y MCE.

Tabla 9. Coeficientes de roce del Aislador FPT8836/14-12/10-7.

\begin{tabular}{|c|c|c|c|c|}
\hline \multirow{2}{*}{$\begin{array}{c}\text { COEFICIENTES } \\
\text { DE ROCE }\end{array}$} & \multicolumn{2}{|c|}{ SISMO DBE } & \multicolumn{2}{c|}{ SISMO MCE } \\
\cline { 2 - 5 } SD1 = .6032 & \multicolumn{2}{c|}{ SD1 = 1.5 * 0.6032 } \\
\hline Máximos & $\mu 1=0.0685$ & $\mu 2=0.0157$ & $\mu 1=0.0685$ & $\mu 2=0.0157$ \\
\hline Nominales & $\mu 1=0.0608$ & $\mu 2=0.0124$ & $\mu 1=0.0608$ & $\mu 2=0.0124$ \\
\hline Mínimos & $\mu 1=0.0547$ & $\mu 2=0.0099$ & $\mu 1=0.0547$ & $\mu 2=0.0099$ \\
\hline
\end{tabular}




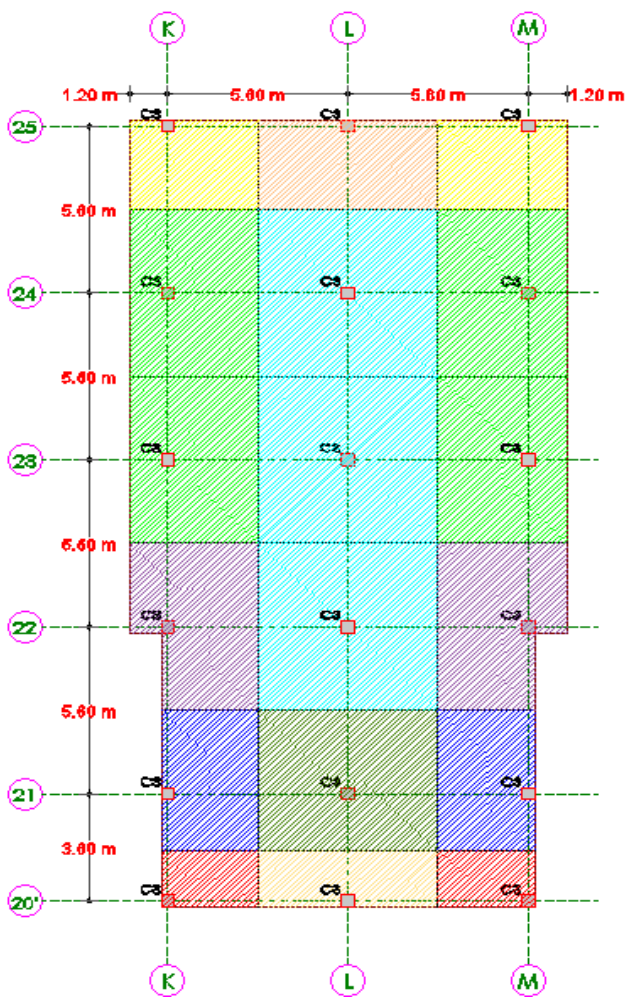

Planta Modelo 1

Estructura Flexible

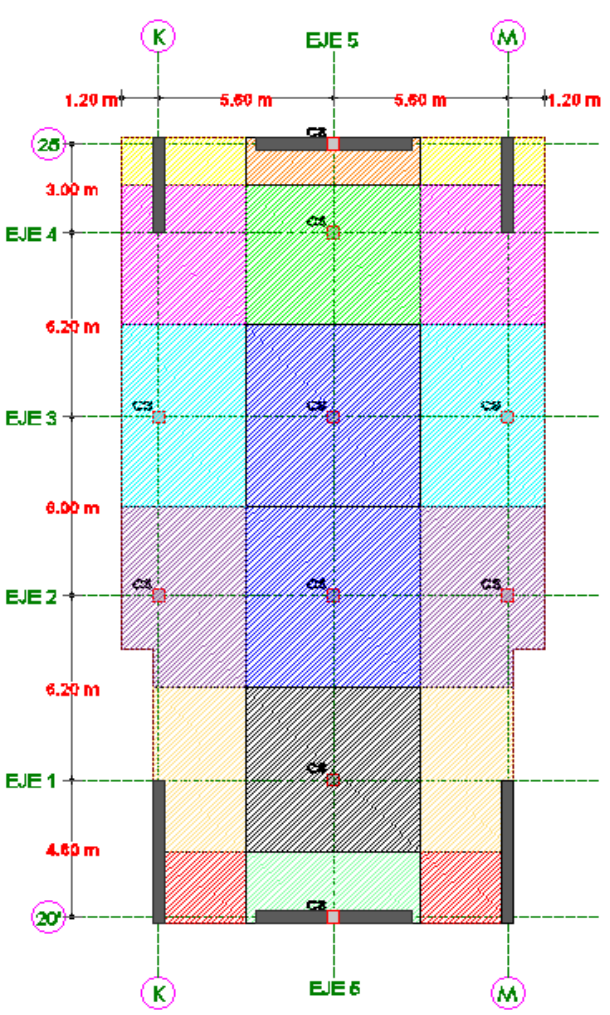

Planta Modelo 2

Estructura Rígida

Figura 11. Mosaico de cargas del modelo 1 y 2 para determinar la carga vertical que llega a cada aislador FPT.

\section{ESTRUCTURA DE ANÁLISIS (BLOQUE I ZE)}

En la figura 12, se presenta uno de los bloques estructurales del nuevo edificio: "Vinculación con la Sociedad" perteneciente a la Universidad de Fuerzas Armadas ESPE - sede Santo Domingo, asignado como bloque estructural I ZE, con aisladores FPT8836/14-12/10-7 en la base; por lo tanto para el análisis sísmico se plantean dos alternativas de cálculo denominadas como estructuras aisladas con y sin muros de corte.

En la figura 13, se indica la planta como Estructura Flexible inscrita como modelo 1, la planta como Estructura Rígida inscrita como modelo 2 y en 3D las dos estructuras modeladas en el programa SAP2000 para obtener una mejor perspectiva visual de los dos modelos calculados. La planta que conforma tanto la Estructura Flexible como la Estructura Rígida para cada modelo, es regular en planta y en elevación, con 5 pisos de altura más una losa de aislación por la implementación de los aisladores, teniendo un total de 6 pisos para el diseño sismo resistente. 


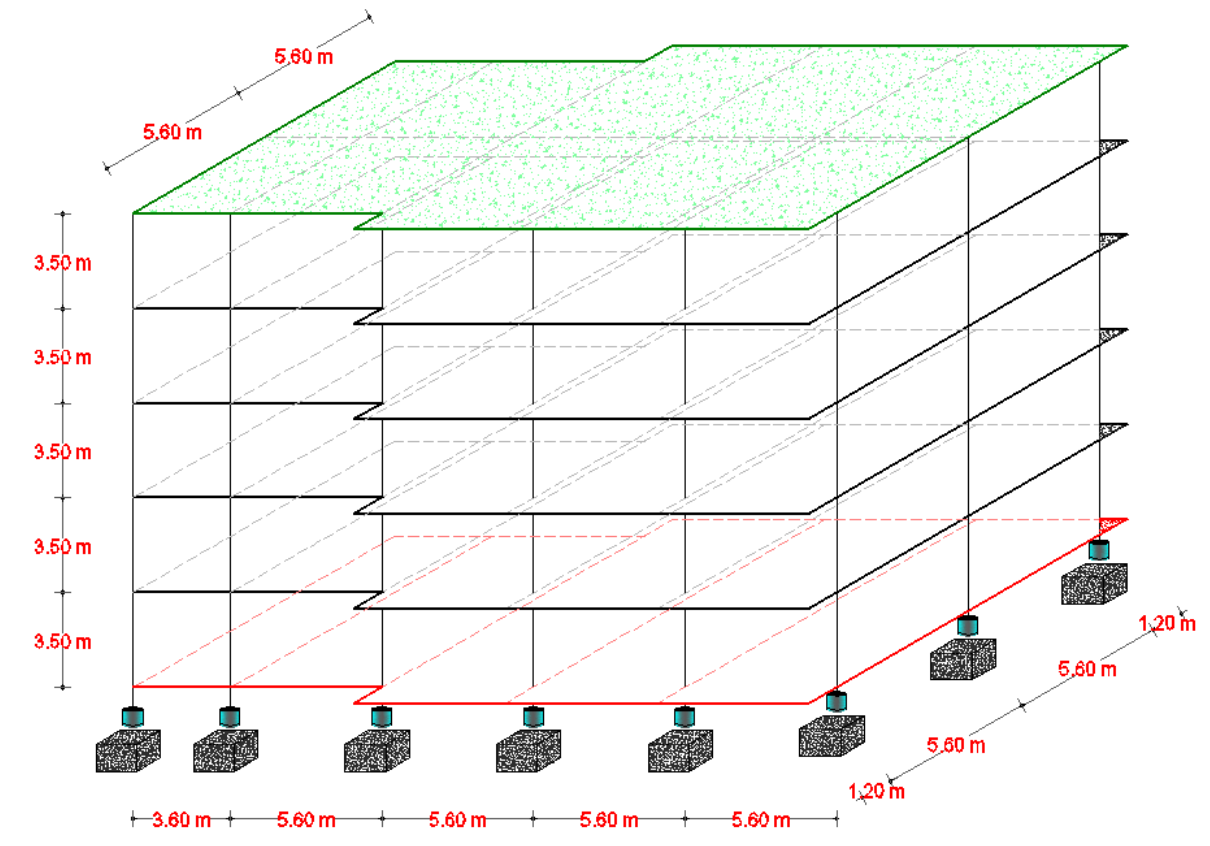

Figura 12. Estructura de análisis sísmico (Bloque I ZE).

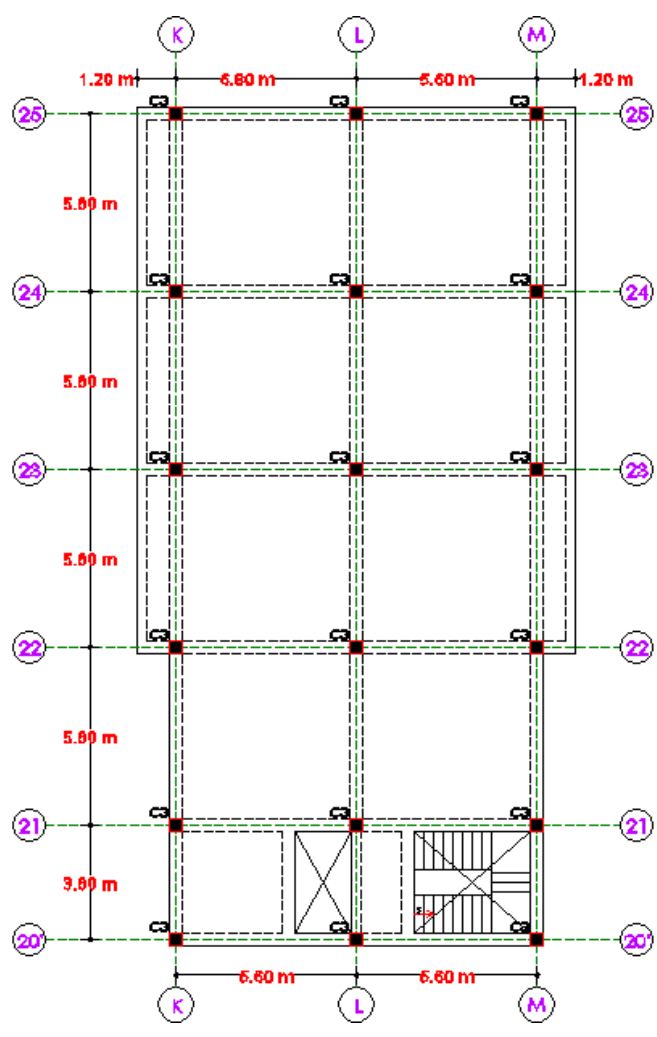

Losa Flexible Nivel $0.00-18.40$ MODELO 1

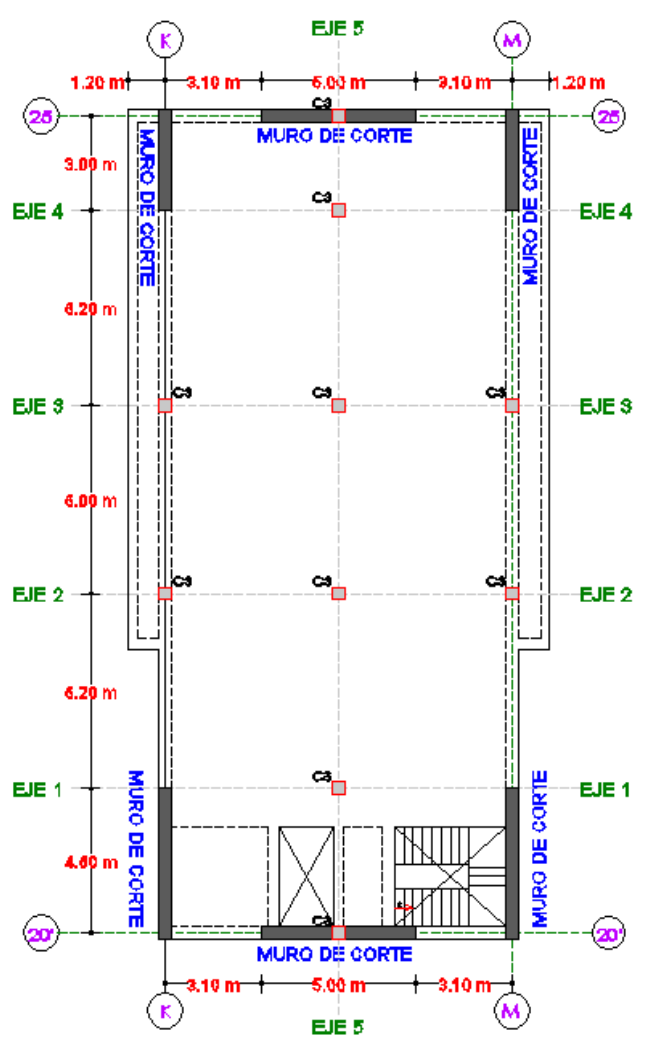

Losa Rígida Nivel 0.00 - 18.40 MODELO 2 


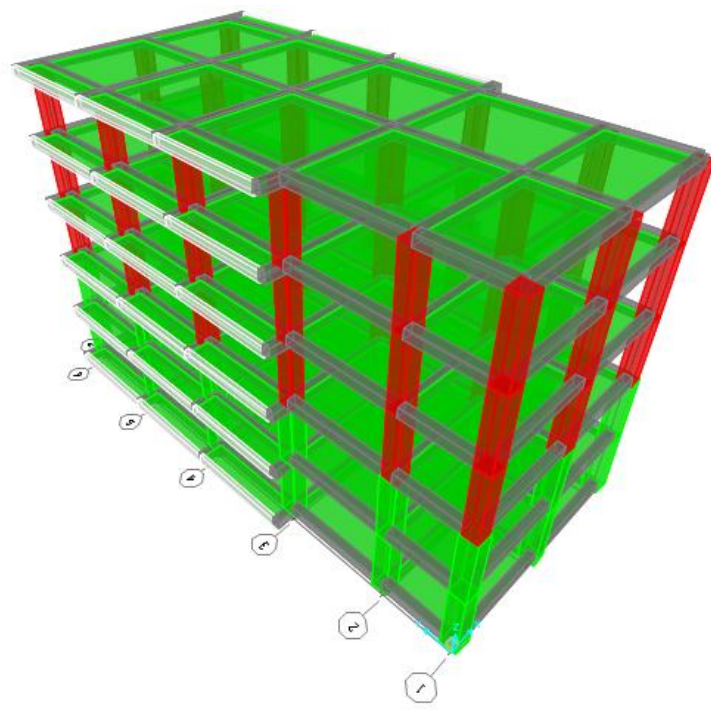

Estructura Flexible

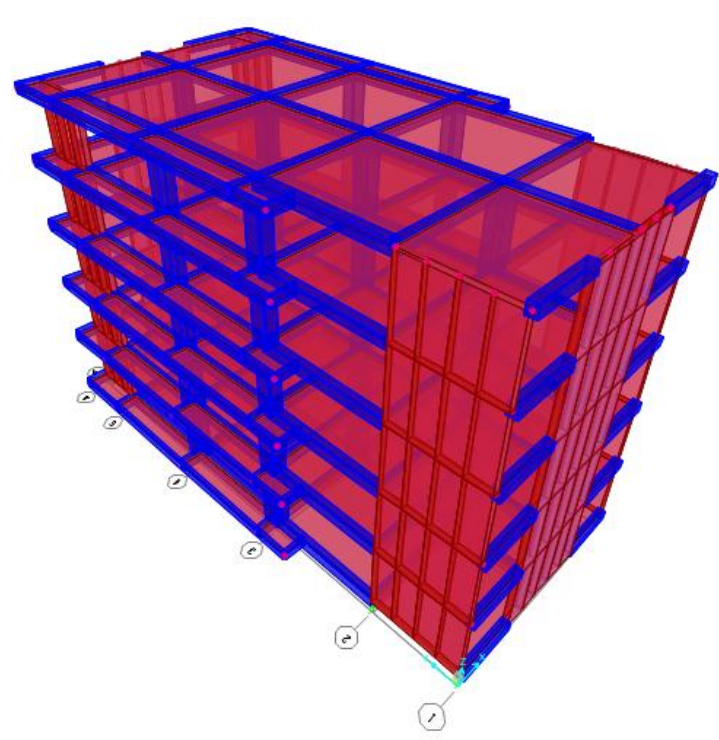

Estructura Rígida

Vista en 3D - SAP2000

Figura 13. Estructuras (Modelo 1 y 2), e Isometría de las losas Flexible y Rígida de la UFA - ESPE, campus Santo Domingo.

Inicialmente se calculó tanto la Estructura Rígida como la Estructura Flexible aplicando el Método de Superposición Modal con las siguientes condiciones: para el modelo 1 y modelo 2 se planteó el análisis sísmico espacial con columnas de hormigón armado siendo para el primero y segundo piso (80×80) $\mathrm{cm}$ y a partir del tercero en adelante columnas de $(70 \times 70) \mathrm{cm}$, con vigas descolgadas de $(40 \times 50) \mathrm{cm}$ más aisladores sísmicos FPT en la base, adicionalmente para el modelo 2 se trabajó con muros de corte con $30 \mathrm{~cm}$ de espesor.

Posterior al cálculo mencionado, con las mismas condiciones estructurales para cada modelo propuesto, se trabajó con el Método Simplificado Lineal del ASCE 7-10 por ser una estructura regular tanto en planta como en elevación, tomando en cuenta para éste método los sismos DBE y MCE.

\section{SISMOS DE ANÁLISIS}

El espectro de diseño DBE, es el que reporta la Norma Ecuatoriana de la Construcción (NEC-15), para éste caso de aplicación es un perfil de suelo tipo $D$ con un factor de peligrosidad sísmica $Z=0.4$ correspondientes a los factores de sitio $F_{a}=1.2 ; F_{d}=1.19 ; F_{s}=1.28$ y el espectro máximo considerado MCE se halla multiplicando el espectro de diseño por un factor de 1.5 (ASCE 7-10). 
Se consideró un factor de reducción de fuerzas sísmicas $R=1$; un factor de irregularidad en planta $\emptyset_{p}=1$ y un factor de irregularidad en elevación $\emptyset_{e}=1$; por lo tanto, es el espectro elástico para el $5 \%$ de amortiguamiento el mismo que se divide para el valor de $B$. Ver ecuación 11.

La ecuación (11) se utilizó para el Método de Superposición Modal y de igual forma para el espectro cargado en el modelamiento de las dos estructuras en SAP2000; en cambio para el Método Simplificado Lineal se trabajó con los valores de $B$ indicados en la tabla 2 y con un $R=1$.

\section{RESULTADOS}

Se realizó un análisis sísmico espacial, para cada una de las estructuras propuestas (modelo 1 y modelo 2 ) como se observa en la figura 13. Los resultados expuestos se encuentran unificados al S.I. de medidas aplicando la siguiente equivalencia: 1 Tonf $=9.8067 \mathrm{KN}$.

Por otra parte, los desplazamientos que se hallan para el Método Simplificado Lineal $D_{D}$, fueron multiplicados por 1.1 para tomar en cuenta los efectos de torsión natural y accidental; de igual manera se indica que, el periodo calculado es el que se obtiene en el sistema de aislación. Ver ecuación 4.

\subsection{ANÁLISIS DE ESTRUCTURA FLEXIBLE}

Se presenta los resultados obtenidos con los tres modelos matemáticos planteados, Método Espectral (3 fases), Simplificado Lineal y SAP2000; aplicados para cada sismo DBE y MCE en: periodos, desplazamientos, fuerzas laterales y derivas en el centro de masas de cada piso. Para el análisis sísmico espectral se trabajó con 18 grados de libertad; es decir, se consideran tres grados de libertad por planta: dos componentes de desplazamiento horizontal y una rotación de piso.

\subsubsection{Resultados del análisis sísmico en sentido $X$}

Tabla 10. Periodos calculados para sismo DBE y MCE con Método Espectral, Simplificado Lineal y SAP2000 para Estructura Flexible.

\begin{tabular}{|c|c|c|c|c|c|c|}
\hline \multirow{2}{*}{$\begin{array}{c}\text { BLOQUE } \\
\text { I ZE }\end{array}$} & \multicolumn{5}{|c|}{ PERIODOS } \\
\cline { 2 - 7 } $\begin{array}{c}\text { SENTIDO } \\
\text { "X" }\end{array}$ & $\begin{array}{c}\text { Modal- } \\
\text { Espectral } \\
\text { (3 Fases) }\end{array}$ & ASCE 7-10 & SAP2000 & $\begin{array}{c}\text { Modal- } \\
\text { Espectral } \\
\text { (3 Fases) }\end{array}$ & ASCE 7-10 & SAP2000 \\
\hline T1 (seg) & 2.9672 & 2.8614 & 2.9180 & 3.3961 & 3.2971 & 3.3417 \\
\hline T2 (seg) & 2.9466 & 2.8614 & 2.8890 & 3.3783 & 3.2971 & 3.3167 \\
\hline T3 (seg) & 2.8546 & 2.8614 & 2.6836 & 3.2727 & 3.2971 & 3.0866 \\
\hline
\end{tabular}


Tabla 11. Desplazamientos calculados para sismo DBE y MCE con Método Espectral, Simplificado Lineal y SAP2000 para Estructura Flexible.

\begin{tabular}{|c|c|c|c|c|c|c|}
\hline \multirow{2}{*}{$\begin{array}{c}\text { BLOQUE } \\
\text { I ZE }\end{array}$} & \multicolumn{5}{|c|}{ DESPLAZAMIENTOS } \\
\cline { 2 - 7 } & \multicolumn{3}{|c|}{ SISMO DBE } & \multicolumn{3}{c|}{ SISMO MCE } \\
\hline $\begin{array}{c}\text { SENTIDO } \\
\text { "X" }\end{array}$ & $\begin{array}{c}\text { Modal- } \\
\text { Espectral } \\
\text { (3 Fases) }\end{array}$ & ASCE 7-10 & SAP2000 & $\begin{array}{c}\text { Modal- } \\
\text { Espectral } \\
\text { (3 Fases) }\end{array}$ & ASCE 7-10 & SAP2000 \\
\hline q ais (cm) & 24.4553 & 24.8044 & 23.1193 & 46.9697 & 47.4137 & 44.7183 \\
\hline q1 (cm) & 25.0975 & 24.8044 & 23.8228 & 47.8933 & 47.4137 & 45.7366 \\
\hline q2 (cm) & 25.7115 & 24.8044 & 24.4952 & 48.7747 & 47.4137 & 46.7087 \\
\hline q3 (cm) & 26.2356 & 24.8044 & 25.0686 & 49.5250 & 47.4137 & 47.5363 \\
\hline q4 (cm) & 26.6058 & 24.8044 & 25.4731 & 50.0534 & 47.4137 & 48.1189 \\
\hline q5 (cm) & 26.8414 & 24.8044 & 25.7301 & 50.3891 & 47.4137 & 48.4887 \\
\hline
\end{tabular}
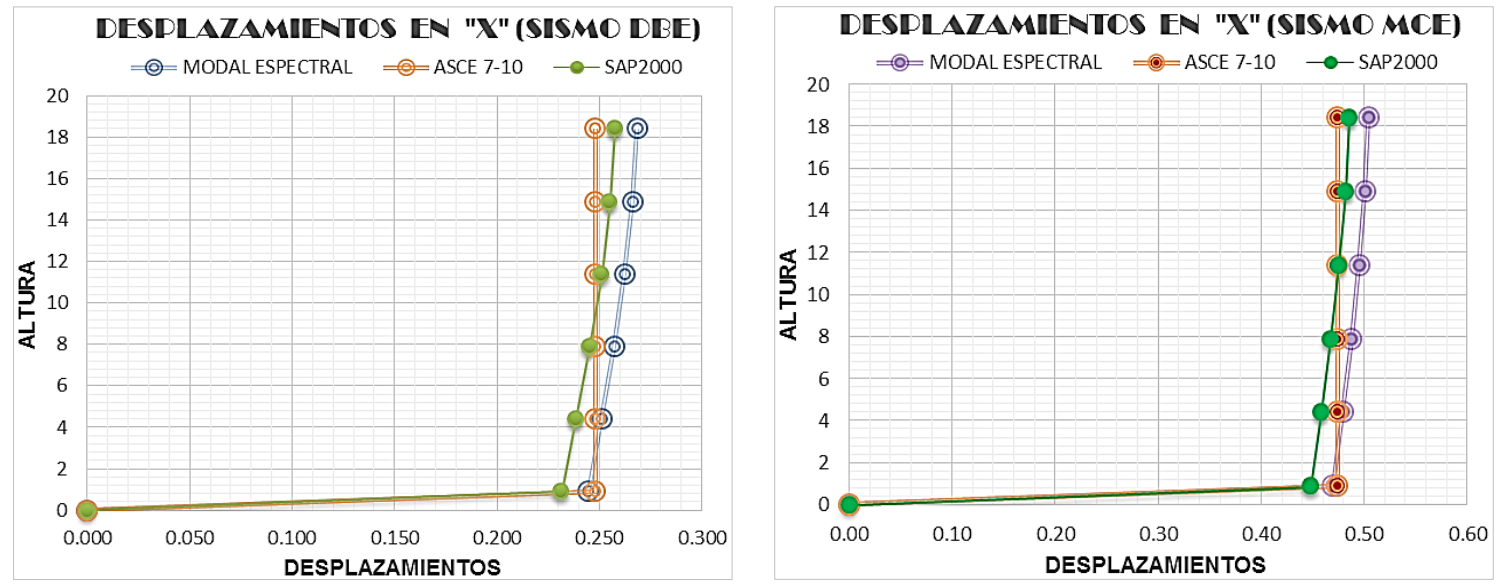

Figura 14. Desplazamientos laterales con Método Espectral, Simplificado Lineal del ASCE 7-10 y SAP2000 para Estructura Flexible.

Tabla 12. Fuerzas Laterales calculadas para sismo DBE y MCE con Método Espectral, Simplificado Lineal y SAP2000 para Estructura Flexible.

\begin{tabular}{|c|c|c|c|c|c|c|}
\hline \multirow{2}{*}{$\begin{array}{c}\text { BLOQUE } \\
\text { I ZE }\end{array}$} & \multicolumn{5}{|c|}{ FUERZAS LATERALES } \\
\cline { 2 - 7 } & \multicolumn{3}{|c|}{ SISMO DBE } & \multicolumn{3}{c|}{ SISMO MCE } \\
\hline $\begin{array}{c}\text { SENTIDO } \\
\text { "X" }\end{array}$ & $\begin{array}{c}\text { Modal- } \\
\text { Espectral } \\
\text { (3 Fases) }\end{array}$ & ASCE 7-10 & SAP2000 & $\begin{array}{c}\text { Modal- } \\
\text { Espectral } \\
\text { (3 Fases) }\end{array}$ & ASCE 7-10 & SAP2000 \\
\hline Fais (KN) & 343.1757 & 335.6881 & 332.3883 & 512.1402 & 483.2841 & 491.5461 \\
\hline F1 (KN) & 317.0859 & 335.6881 & 305.3179 & 470.9373 & 483.2841 & 450.3433 \\
\hline F2 (KN) & 328.4332 & 335.6881 & 316.6652 & 481.3148 & 483.2841 & 460.7207 \\
\hline F3 (KN) & 344.2122 & 335.6881 & 332.4442 & 495.4355 & 483.2841 & 474.8414 \\
\hline F4 (KN) & 357.5170 & 335.6881 & 345.7489 & 506.5621 & 483.2841 & 485.9681 \\
\hline F5 (KN) & 237.4016 & 335.6881 & 225.6335 & 324.5704 & 483.2841 & 303.9763 \\
\hline
\end{tabular}


FUIDZAS LATIRAIIS FN "X" (SISMO DBE)

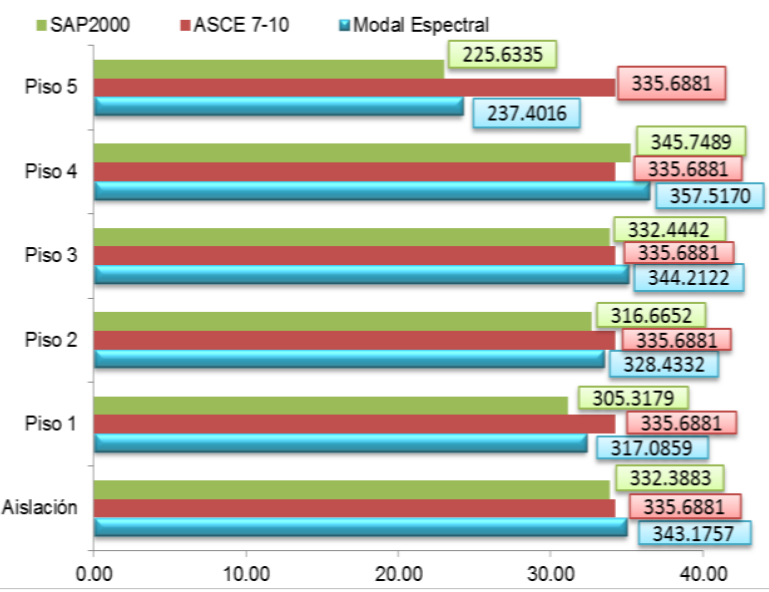

FUERTAS LATERAIES EN "X" (SISMO MCE)

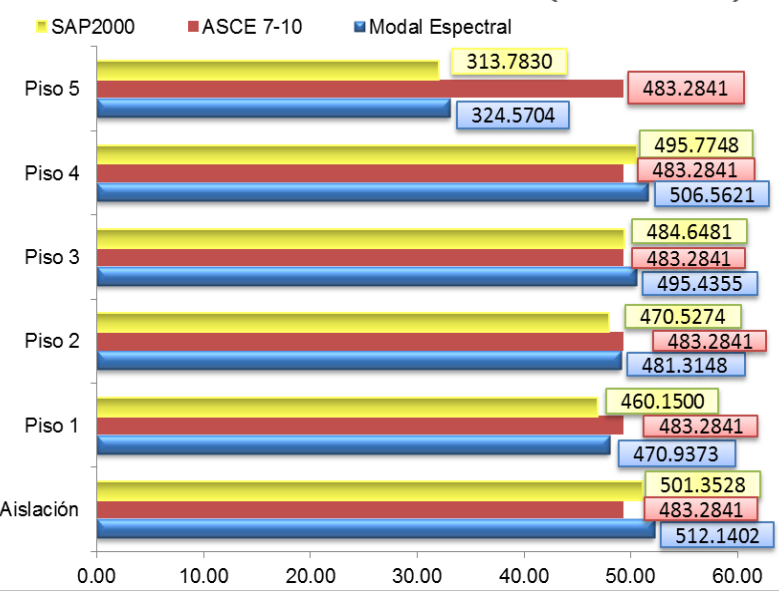

Figura 15. Fuerzas Laterales con Método Espectral,

Simplificado Lineal del ASCE 7-10 y SAP2000 para Estructura Flexible.

Tabla 13. Derivas de Piso calculadas para sismo DBE y MCE con Método Espectral y SAP2000 para Estructura Flexible.

\begin{tabular}{|c|c|c|c|c|}
\hline \multirow{2}{*}{$\begin{array}{c}\text { BLOQUE } \\
\text { I ZE }\end{array}$} & \multicolumn{4}{|c|}{ DERIVAS DE PISO } \\
\cline { 2 - 5 } $\begin{array}{c}\text { SENTIDO } \\
\text { "X" }\end{array}$ & $\begin{array}{c}\text { Modal-Espectral } \\
\text { (3 Fases) }\end{array}$ & SAP2000 & $\begin{array}{c}\text { Modal-Espectral } \\
\text { (3 Fases) }\end{array}$ & SAP2000 \\
\hline Derv ais & 0.0699 & 0.0661 & 0.1342 & 0.1278 \\
\hline Derv1 & 0.0018 & 0.0020 & 0.0026 & 0.0029 \\
\hline Derv2 & 0.0017 & 0.0019 & 0.0025 & 0.0028 \\
\hline Derv3 & 0.0015 & 0.0016 & 0.0021 & 0.0024 \\
\hline Derv4 & 0.0011 & 0.0012 & 0.0015 & 0.0017 \\
\hline Derv5 & 0.0007 & 0.0007 & 0.0010 & 0.0011 \\
\hline
\end{tabular}

DERIVAS SENTIDO "X" (SISMO DBE)

$\multimap$ MODALESPECTRAL $\quad \square-$ SAP2000

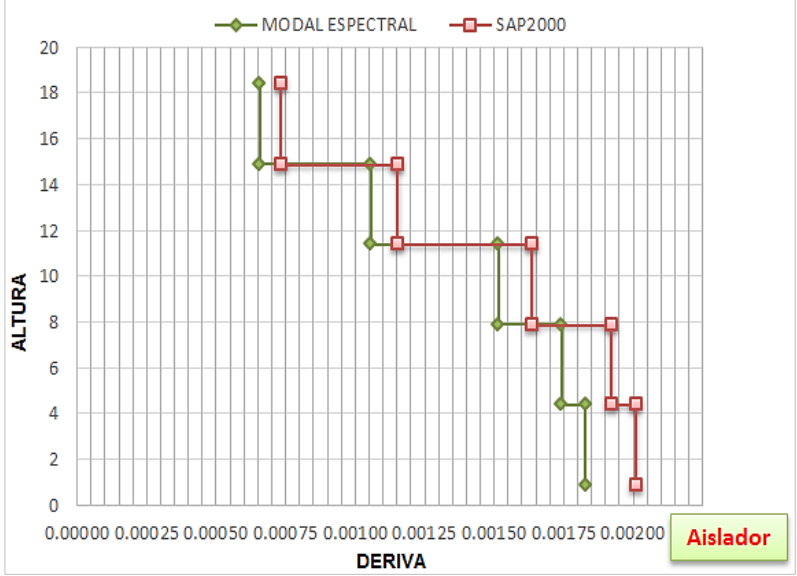

DERIVAS SENTIDO "X" (SISMO MCE)

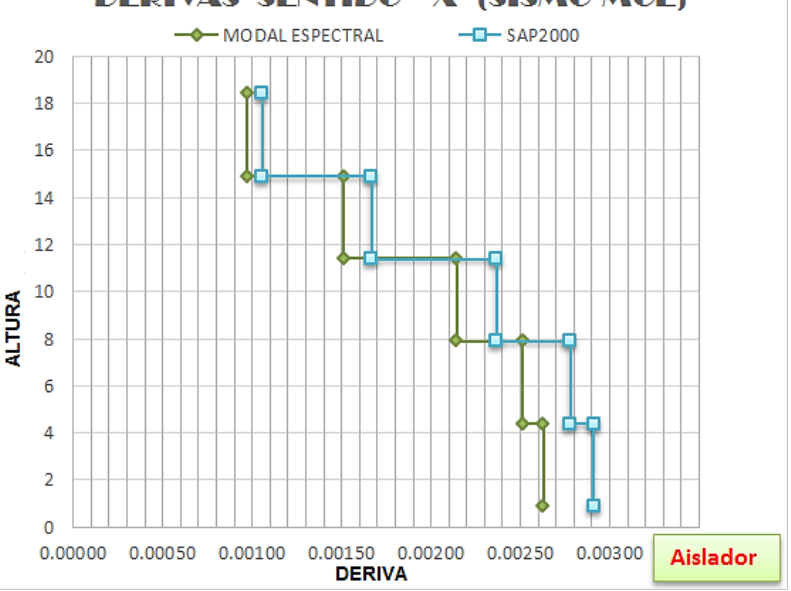

Figura 16. Derivas de piso con Método Espectral y SAP2000 para Estructura Flexible. 


\subsubsection{Resultados del análisis sísmico en sentido $Y$}

En la figuras 14, 15 y 16 se indica: desplazamientos, fuerzas laterales y derivas de pisos para el sentido de análisis " $X$ ", por lo tanto, la estructura flexible tiene un mejor comportamiento en ésta dirección, dando lugar a que "Y" sea el sentido más crítico.

Tabla 14. Desplazamientos calculados para sismo DBE y MCE con Método Espectral, Simplificado Lineal y SAP2000 para Estructura Flexible.

\begin{tabular}{|c|c|c|c|c|c|c|}
\hline \multirow{2}{*}{$\begin{array}{c}\text { BLOQUE } \\
\text { I ZE }\end{array}$} & \multicolumn{6}{|c|}{ DESPLAZAMIENTOS } \\
\cline { 2 - 7 } $\begin{array}{c}\text { SENTIDO } \\
\text { "Y" }\end{array}$ & $\begin{array}{c}\text { Modal- } \\
\text { Espectral } \\
\text { (3 Fases) }\end{array}$ & ASCE 7-10 & SAP2000 & $\begin{array}{c}\text { Modal- } \\
\text { Espectral } \\
\text { (3 Fases) }\end{array}$ & ASCE 7-10 & SAP2000 \\
\hline q ais (cm) & 24.6267 & 24.8044 & 23.3350 & 47.2039 & 47.4137 & 45.0097 \\
\hline q1 (cm) & 25.1060 & 24.8044 & 23.8357 & 47.8956 & 47.4137 & 45.7330 \\
\hline q2 (cm) & 25.5635 & 24.8044 & 24.3146 & 48.5557 & 47.4137 & 46.4241 \\
\hline q3 (cm) & 25.9518 & 24.8044 & 24.7219 & 49.1160 & 47.4137 & 47.0111 \\
\hline q4 (cm) & 26.2182 & 24.8044 & 25.0005 & 49.5003 & 47.4137 & 47.4122 \\
\hline q5 (cm) & 26.3778 & 24.8044 & 25.1656 & 49.7306 & 47.4137 & 47.6496 \\
\hline
\end{tabular}

Tabla 15. Fuerzas Laterales calculadas para sismo DBE y MCE con Método Espectral, Simplificado Lineal y SAP2000 para Estructura Flexible.

\begin{tabular}{|c|c|c|c|c|c|c|}
\hline \multirow{2}{*}{$\begin{array}{c}\text { BLOQUE } \\
\text { I ZE }\end{array}$} & \multicolumn{5}{|c|}{ FUERZAS LATERALES } \\
\cline { 2 - 7 } $\begin{array}{c}\text { SENTIDO } \\
\text { "Y" }\end{array}$ & $\begin{array}{c}\text { Modal- } \\
\text { Espectral } \\
\text { (3 Fases) }\end{array}$ & ASCE 7-10 & SAP2000 & $\begin{array}{c}\text { Modal- } \\
\text { Espectral } \\
\text { (3 Fases) }\end{array}$ & ASCE 7-10 & SAP2000 \\
\hline Fais (KN) & 363.4530 & 335.6881 & 338.9362 & 527.7838 & 483.2841 & 503.2671 \\
\hline F1 (KN) & 332.7747 & 335.6881 & 308.2579 & 483.5998 & 483.2841 & 459.0830 \\
\hline F2 (KN) & 337.1083 & 335.6881 & 312.5915 & 489.4269 & 483.2841 & 464.9101 \\
\hline F3 (KN) & 344.1632 & 335.6881 & 319.6464 & 497.6145 & 483.2841 & 473.0978 \\
\hline F4 (KN) & 353.1000 & 335.6881 & 328.5833 & 506.7936 & 483.2841 & 482.2768 \\
\hline F5 (KN) & 264.7191 & 335.6881 & 240.2024 & 376.8852 & 483.2841 & 352.3685 \\
\hline
\end{tabular}

Tabla 16. Derivas de Piso calculadas para sismo DBE y MCE con Método Espectral y SAP2000 para Estructura Flexible.

\begin{tabular}{|c|c|c|c|c|}
\hline \multirow{2}{*}{$\begin{array}{c}\text { BLOQUE } \\
\text { I ZE }\end{array}$} & \multicolumn{4}{|c|}{ DERIVAS DE PISO } \\
\cline { 2 - 5 } $\begin{array}{c}\text { SENTIDO } \\
\text { "Y" }\end{array}$ & $\begin{array}{c}\text { Modal-Espectral } \\
\text { (3 Fases) }\end{array}$ & SAP2000 & $\begin{array}{c}\text { Modal-Espectral } \\
\text { (3 Fases) }\end{array}$ & SAP2000 \\
\hline Derv ais & 0.0704 & 0.0667 & 0.1349 & 0.1286 \\
\hline Derv1 & 0.0014 & 0.0014 & 0.0020 & 0.0021 \\
\hline Derv2 & 0.0013 & 0.0014 & 0.0019 & 0.0020 \\
\hline Derv3 & 0.0011 & 0.0012 & 0.0016 & 0.0017 \\
\hline Derv4 & 0.0008 & 0.0008 & 0.0011 & 0.0011 \\
\hline Derv5 & 0.0005 & 0.0005 & 0.0007 & 0.0007 \\
\hline
\end{tabular}




\subsection{ANÁLISIS DE ESTRUCTURA RÍGIDA}

Para la Estructura Rígida se tomó la misma configuración para el reporte de resultados con las tres metodologías sísmicas de cálculo: Espectral, Simplificado Lineal y SAP2000 para DBE y MCE como se indica.

\subsubsection{Resultados del análisis sísmico en sentido $X$}

Tabla 17. Periodos calculados para sismo DBE y MCE con Método Espectral, Simplificado Lineal y SAP2000 para Estructura Rígida.

\begin{tabular}{|c|c|c|c|c|c|c|}
\hline \multirow{2}{*}{$\begin{array}{c}\text { BLOQUE } \\
\text { I ZE }\end{array}$} & \multicolumn{5}{|c|}{ PERIODOS } \\
\cline { 2 - 7 } $\begin{array}{c}\text { SENTIDO } \\
\text { "X" }\end{array}$ & $\begin{array}{c}\text { Modal- } \\
\text { Espectral } \\
\text { (3 Fases) }\end{array}$ & ASCE 7-10 & SAP2000 & $\begin{array}{c}\text { Modal- } \\
\text { Espectral } \\
\text { (3 Fases) }\end{array}$ & ASCE 7-10 & SAP2000 \\
\hline T1 (seg) & 3.3507 & 2.8614 & 3.2155 & 3.8471 & 3.2971 & 3.7115 \\
\hline T2 (seg) & 3.0621 & 2.8614 & 3.1281 & 3.5002 & 3.2971 & 3.6057 \\
\hline T3 (seg) & 2.8012 & 2.8614 & 3.1148 & 3.2210 & 3.2971 & 3.5911 \\
\hline
\end{tabular}

Tabla 18. Desplazamientos calculados para sismo DBE y MCE con Método Espectral, Simplificado Lineal y SAP2000 para Estructura Rígida.

\begin{tabular}{|c|c|c|c|c|c|c|}
\hline \multirow{2}{*}{$\begin{array}{c}\text { BLOQUE } \\
\text { I ZE }\end{array}$} & \multicolumn{5}{|c|}{ SISMO DBE } & \multicolumn{3}{c|}{ SISMO MCE } \\
\cline { 2 - 7 } $\begin{array}{c}\text { SENTIDO } \\
\text { "X" }\end{array}$ & $\begin{array}{c}\text { Modal- } \\
\text { Espectral } \\
\text { (3 Fases) }\end{array}$ & ASCE 7-10 & SAP2000 & $\begin{array}{c}\text { Modal- } \\
\text { Espectral } \\
\text { (3 Fases) }\end{array}$ & ASCE 7-10 & SAP2000 \\
\hline q ais (cm) & 25.8251 & 24.8044 & 25.6689 & 48.6838 & 47.4137 & 49.4580 \\
\hline q1 (cm) & 26.1464 & 24.8044 & 25.7469 & 49.1344 & 47.4137 & 49.5705 \\
\hline q2 (cm) & 26.4492 & 24.8044 & 25.8916 & 49.5586 & 47.4137 & 49.7794 \\
\hline q3 (cm) & 26.7209 & 24.8044 & 26.0553 & 49.9389 & 47.4137 & 50.0157 \\
\hline q4 (cm) & 26.9578 & 24.8044 & 26.2226 & 50.2702 & 47.4137 & 50.2569 \\
\hline q5 (cm) & 27.1683 & 24.8044 & 26.3811 & 50.5643 & 47.4137 & 50.4855 \\
\hline
\end{tabular}
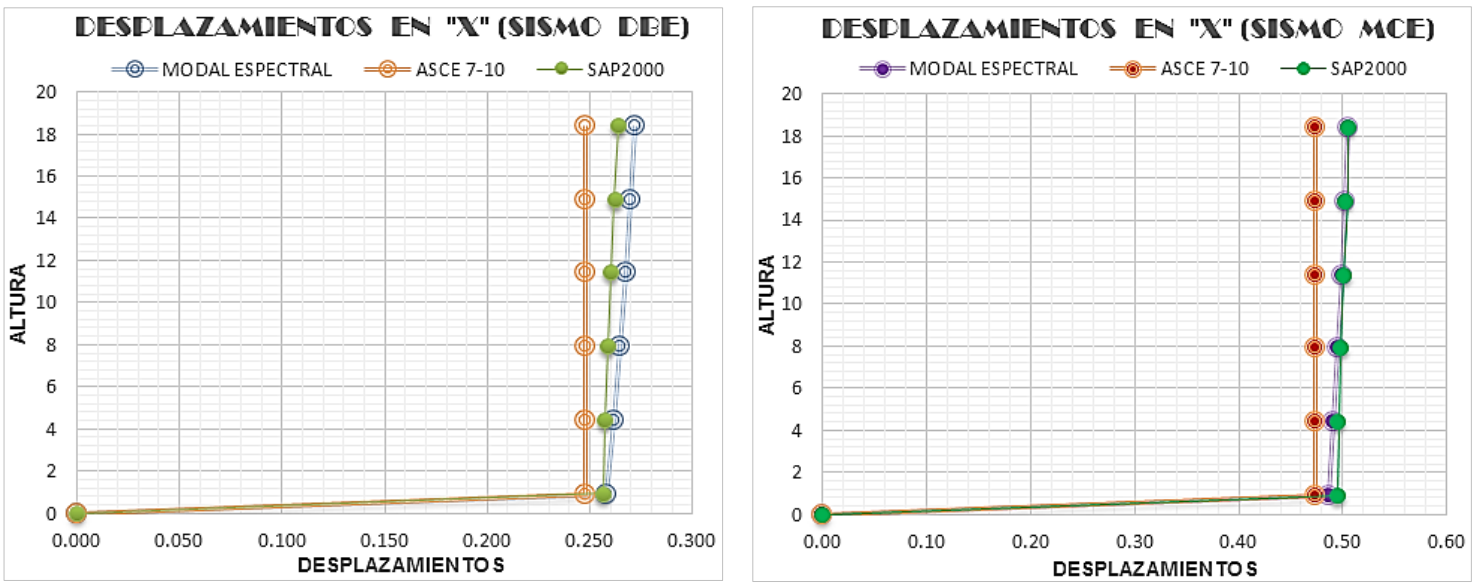

Figura 17. Desplazamientos laterales con Método Espectral, Simplificado Lineal del ASCE 7-10 y SAP2000 para Estructura Rígida. 
Tabla 19. Fuerzas laterales calculadas para sismo DBE y MCE con Método Espectral, Simplificado Lineal y SAP2000 para Estructura Rígida.

\begin{tabular}{|c|c|c|c|c|c|c|}
\hline \multirow{2}{*}{$\begin{array}{c}\text { BLOQUE } \\
\text { I ZE }\end{array}$} & \multicolumn{5}{|c|}{ FUERZAS LATERALES } \\
\cline { 2 - 7 } $\begin{array}{c}\text { SENTIDO } \\
\text { "X” }\end{array}$ & $\begin{array}{c}\text { Modal- } \\
\text { Espectral } \\
\text { (3 Fases) }\end{array}$ & ASCE 7-10 & SAP2000 & $\begin{array}{c}\text { Modal- } \\
\text { Espectral } \\
\text { (3 Fases) }\end{array}$ & ASCE 7-10 & SAP2000 \\
\hline Fais (KN) & 312.1698 & 388.8462 & 323.0553 & 443.5757 & 559.8030 & 458.2857 \\
\hline F1 (KN) & 281.7465 & 388.8462 & 292.6319 & 393.0643 & 559.8030 & 407.7744 \\
\hline F2 (KN) & 277.2835 & 388.8462 & 288.1689 & 376.6459 & 559.8030 & 391.3560 \\
\hline F3 (KN) & 265.4105 & 388.8462 & 276.2959 & 346.1951 & 559.8030 & 360.9052 \\
\hline F4 (KN) & 236.0129 & 388.8462 & 246.8984 & 287.9110 & 559.8030 & 302.6210 \\
\hline F5 (KN) & 133.5535 & 388.8462 & 144.4390 & 146.7837 & 559.8030 & 161.4938 \\
\hline
\end{tabular}
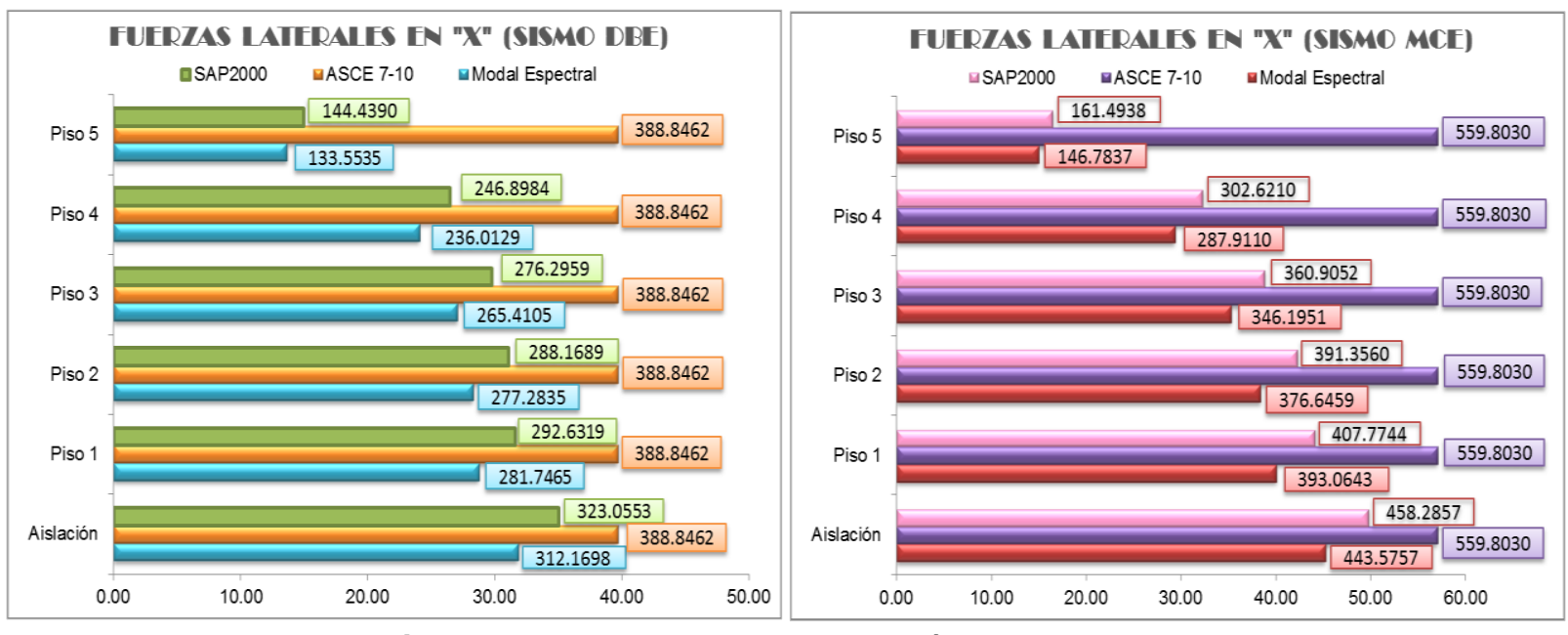

Figura 18. Fuerzas Laterales con Método Espectral,

Simplificado Lineal del ASCE 7-10 y SAP2000 para Estructura Rígida.

Tabla 20. Derivas de piso calculadas para sismo DBE y MCE con Método Espectral y SAP2000 para Estructura Rígida.

\begin{tabular}{|c|c|c|c|c|}
\hline \multirow{2}{*}{$\begin{array}{c}\text { BLOQUE } \\
\text { I ZE }\end{array}$} & \multicolumn{3}{|c|}{ DERIVAS } \\
\cline { 2 - 5 } $\begin{array}{c}\text { SENTIDO } \\
\text { "X" }\end{array}$ & $\begin{array}{c}\text { SISMO DBE } \\
\begin{array}{c}\text { Mspectral- } \\
\text { (3 Fases) }\end{array}\end{array}$ & SAP2000 & $\begin{array}{c}\text { Modal- } \\
\text { Espectral } \\
\text { (3 Fases) }\end{array}$ & SAP2000 \\
\hline Derv ais & 0.0738 & 0.0733 & 0.1391 & 0.1413 \\
\hline Derv1 & 0.0009 & 0.0009 & 0.0013 & 0.0014 \\
\hline Derv2 & 0.0009 & 0.0008 & 0.0012 & 0.0013 \\
\hline Derv3 & 0.0008 & 0.0007 & 0.0011 & 0.0012 \\
\hline Derv4 & 0.0007 & 0.0007 & 0.0009 & 0.0010 \\
\hline Derv5 & 0.0006 & 0.0006 & 0.0008 & 0.0009 \\
\hline
\end{tabular}



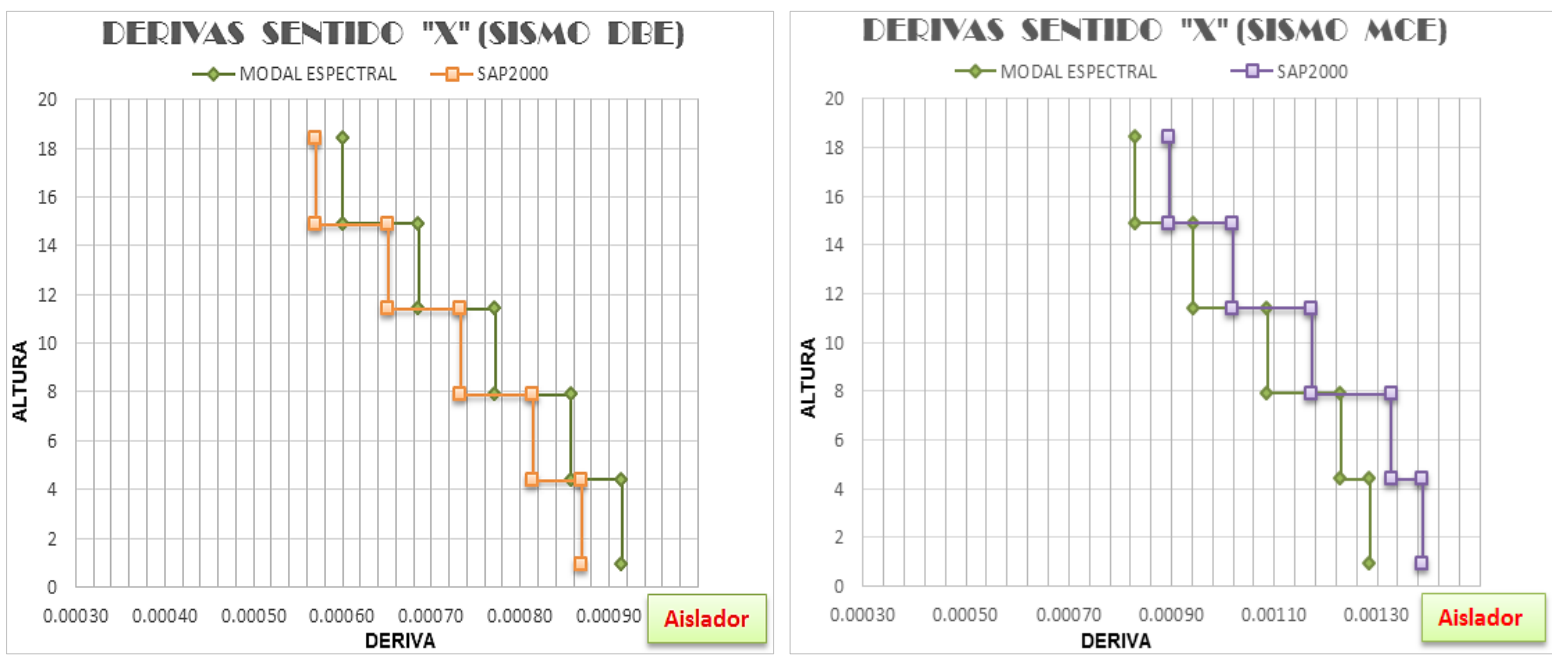

Figura 19. Derivas de Piso con Método Espectral y SAP2000 para Estructura Rígida.

\subsubsection{Resultados del análisis sísmico en sentido $Y$}

En la figuras 17, 18 y 19 se indica: desplazamientos, fuerzas laterales y derivas de pisos para el sentido de análisis " $X$ ", por lo tanto, la estructura rígida tiene un mejor comportamiento en ésta dirección, dando lugar a que " $Y$ " sea el sentido más crítico.

Tabla 21. Desplazamientos calculados para sismo DBE y MCE con Método Espectral, Simplificado Lineal y SAP2000 para Estructura Rígida.

\begin{tabular}{|c|c|c|c|c|c|c|}
\hline \multirow{2}{*}{$\begin{array}{c}\text { BLOQUE } \\
\text { I ZE }\end{array}$} & \multicolumn{5}{|c|}{ DESPLAZAMIENTOS } \\
\cline { 2 - 7 } $\begin{array}{c}\text { SENTIDO } \\
\text { "Y" }\end{array}$ & $\begin{array}{c}\text { Modal- } \\
\text { Espectral } \\
\text { (3 Fases) }\end{array}$ & ASCE 7-10 & SAP2000 & $\begin{array}{c}\text { Modal- } \\
\text { Espectral } \\
\text { (3 Fases) }\end{array}$ & ASCE 7-10 & SAP2000 \\
\hline q ais (cm) & 25.7214 & 24.8044 & 25.9696 & 49.4472 & 47.4137 & 50.0495 \\
\hline q1 (cm) & 26.1172 & 24.8044 & 26.0410 & 49.9020 & 47.4137 & 50.1526 \\
\hline q2 (cm) & 26.5004 & 24.8044 & 26.1837 & 50.3355 & 47.4137 & 50.3586 \\
\hline q3 (cm) & 26.8613 & 24.8044 & 26.3516 & 50.6725 & 47.4137 & 50.6010 \\
\hline q4 (cm) & 27.1955 & 24.8044 & 26.5259 & 50.9539 & 47.4137 & 50.8524 \\
\hline q5 (cm) & 27.5055 & 24.8044 & 26.6937 & 51.1277 & 47.4137 & 51.0945 \\
\hline
\end{tabular}


Tabla 22. Fuerzas Laterales calculadas para sismo DBE y MCE con Método Espectral, Simplificado Lineal y SAP2000 para Estructura Rígida.

\begin{tabular}{|c|c|c|c|c|c|c|}
\hline \multirow{2}{*}{$\begin{array}{c}\text { BLOQUE } \\
\text { I ZE }\end{array}$} & \multicolumn{5}{|c|}{ FUERZAS LATERALES } \\
\cline { 2 - 7 } & \multicolumn{3}{|c|}{ SISMO DBE } & \multicolumn{3}{c|}{ SISMO MCE } \\
\hline $\begin{array}{c}\text { SENTIDO } \\
\text { "Y” }\end{array}$ & $\begin{array}{c}\text { Modal- } \\
\text { Espectral } \\
\text { (3 Fases) }\end{array}$ & ASCE 7-10 & SAP2000 & $\begin{array}{c}\text { Modal- } \\
\text { Espectral } \\
\text { (3 Fases) }\end{array}$ & ASCE 7-10 & SAP2000 \\
\hline Fais (KN) & 345.2517 & 388.8462 & 356.1372 & 513.9711 & 559.8030 & 524.8565 \\
\hline F1 (KN) & 317.2948 & 388.8462 & 328.1802 & 470.5814 & 559.8030 & 481.4668 \\
\hline F2 (KN) & 323.4054 & 388.8462 & 334.2908 & 474.5815 & 559.8030 & 485.4670 \\
\hline F3 (KN) & 331.8999 & 388.8462 & 342.7854 & 478.9445 & 559.8030 & 489.8300 \\
\hline F4 (KN) & 342.3852 & 388.8462 & 353.2707 & 484.9560 & 559.8030 & 495.8415 \\
\hline F5 (KN) & 259.3813 & 388.8462 & 270.2668 & 359.0929 & 559.8030 & 369.9784 \\
\hline
\end{tabular}

Tabla 23. Derivas de Piso calculadas para sismo DBE y MCE con Método Espectral y SAP2000 para Estructura Rígida.

\begin{tabular}{|c|c|c|c|c|}
\hline \multirow{2}{*}{$\begin{array}{c}\text { BLOQUE } \\
\text { I ZE }\end{array}$} & \multicolumn{4}{|c|}{ DERIVAS DE PISO } \\
\cline { 2 - 5 } $\begin{array}{c}\text { SENTIDO } \\
\text { "Y" }\end{array}$ & $\begin{array}{c}\text { Modal-Espectral } \\
\text { (3 Fases) }\end{array}$ & SAP2000 & $\begin{array}{c}\text { Modal-Espectral } \\
\text { (3 Fases) }\end{array}$ & SAP2000 \\
\hline Derv ais & 0.0735 & 0.0742 & 0.1413 & 0.1430 \\
\hline Derv1 & 0.0011 & 0.0002 & 0.0013 & 0.0003 \\
\hline Derv2 & 0.0011 & 0.0004 & 0.0013 & 0.0006 \\
\hline Derv3 & 0.0010 & 0.0005 & 0.0009 & 0.0007 \\
\hline Derv4 & 0.0010 & 0.0005 & 0.0008 & 0.0007 \\
\hline Derv5 & 0.0009 & 0.0005 & 0.0005 & 0.0007 \\
\hline
\end{tabular}

\subsection{COMPARACIÓN DE ESTRUCTURA RÍGIDA CON ESTRUCTURA FLEXIBLE}

Finalmente, se presenta en las tablas de la 24 hasta 37 una comparación de resultados obtenidos entre las estructuras Rígida y Flexible mediante el Método de Superposición Modal y el programa de modelación estructural SAP2000 aplicando los sismos $\boldsymbol{D B E}$ y $\boldsymbol{M C E}$, por consiguiente el desempeño de los dos métodos se calcularon en los dos sentidos de análisis " $X$ " $\mathrm{y}$ " $Y$ ".

En la estructura flexible, para el sismo $\boldsymbol{D B E}$ la deriva de piso máxima es el $0.18 \%$ y para el sismo $M C E$ es el $0.26 \%$, valores muy bajos por ser una estructura con aisladores sísmicos. El NEC-15 permite una deriva máxima del $2 \%$ para el sismo DBE, de tal manera que la estructura flexible cumple con esta condición por tener sus derivas muy por debajo del promedio, por lo tanto no implica daño en los elementos estructurales, no estructurales y sus componentes. 
Sin embargo, ésta investigación se basa en la comparación de estructuras aisladas con y sin muros de corte; por lo que se tiene en la estructura rígida una deriva máxima de $0.091 \%$ para el sismo $\boldsymbol{D B E}$ y $0.129 \%$ para $\boldsymbol{M C E}$; valores mucho más bajos a comparación con la estructura flexible, es decir que cumple la función perfecta de aislar la superestructura del suelo y así evitar que se produzcan daños severos o el colapso de la misma ante eventos sísmicos de gran capacidad.

\subsubsection{Resultados comparativos del análisis sísmico en sentido $\mathrm{X}$}

A continuación se presenta la tabulación de resultados en sentido " $X$ " de la comparación entre las dos estructuras Rígida y Flexible en base a los dos métodos estructurales ya mencionados, para el efecto se enfatiza que, en el sentido de análisis "X" se obtuvieron resultados con una mejor conducta sísmica.

Tabla 24. Comparación entre estructuras Rígida y Flexible a nivel de Periodos para sismo DBE en sentido "X". Con Método Espectral y SAP2000.

\begin{tabular}{|c|c|c|c|c|}
\hline BLOQUE I ZE & \multicolumn{4}{|c|}{ PERIODOS } \\
\hline $\begin{array}{c}\text { TIPO DE } \\
\text { ESTRUCTURA }\end{array}$ & FLEXIBLE & RÍGIDA & FLEXIBLE & RÍGIDA \\
\hline $\begin{array}{c}\text { SENTIDO } \\
\text { "X" }\end{array}$ & $\begin{array}{c}\text { MODAL } \\
\text { ESPECTRAL }\end{array}$ & $\begin{array}{c}\text { MODAL } \\
\text { ESPECTRAL }\end{array}$ & SAP2000 & SAP2000 \\
\hline T1 (seg) & 2.9672 & 3.3507 & 2.9180 & 3.2155 \\
\hline T2 (seg) & 2.9466 & 3.0621 & 2.8890 & 3.1281 \\
\hline T3 (seg) & 2.8546 & 2.8012 & 2.6836 & 3.1148 \\
\hline
\end{tabular}

Tabla 25. Comparación entre estructuras Rígida y Flexible a nivel de Desplazamientos para sismo DBE sentido "X". Con Método Espectral y SAP2000.

\begin{tabular}{|c|c|c|c|c|}
\hline BLOQUE I ZE & \multicolumn{4}{|c|}{ DESPLAZAMIENTOS } \\
\hline $\begin{array}{c}\text { TIPO DE } \\
\text { ESTRUCTURA }\end{array}$ & FLEXIBLE & RÍGIDA & FLEXIBLE & RÍGIDA \\
\hline $\begin{array}{c}\text { SENTIDO } \\
\text { "X" }\end{array}$ & $\begin{array}{c}\text { MODAL } \\
\text { ESPECTRAL }\end{array}$ & $\begin{array}{c}\text { MODAL } \\
\text { ESPECTRAL }\end{array}$ & SAP2000 & SAP2000 \\
\hline q ais (cm) & 24.4553 & 25.8251 & 23.1193 & 25.6689 \\
\hline q1 (cm) & 25.0975 & 26.1464 & 23.8228 & 25.7469 \\
\hline q2 (cm) & 25.7115 & 26.4492 & 24.4952 & 25.8916 \\
\hline q3 (cm) & 26.2356 & 26.7209 & 25.0686 & 26.0553 \\
\hline q4 (cm) & 26.6058 & 26.9578 & 25.4731 & 26.2226 \\
\hline q5 (cm) & 26.8414 & 27.1683 & 25.7301 & 26.3811 \\
\hline
\end{tabular}


Tabla 26. Comparación entre estructuras Rígida y Flexible a nivel de Fuerzas para sismo DBE en sentido "X". Con Método Espectral y SAP2000.

\begin{tabular}{|c|c|c|c|c|}
\hline BLOQUE I ZE & \multicolumn{4}{|c|}{ FUERZAS LATERALES } \\
\hline $\begin{array}{c}\text { TIPO DE } \\
\text { ESTRUCTURA }\end{array}$ & FLEXIBLE & RÍGIDA & FLEXIBLE & RÍGIDA \\
\hline $\begin{array}{c}\text { SENTIDO } \\
\text { "X" }\end{array}$ & $\begin{array}{c}\text { MODAL } \\
\text { ESPECTRAL }\end{array}$ & $\begin{array}{c}\text { MODAL } \\
\text { ESPECTRAL }\end{array}$ & SAP2000 & SAP2000 \\
\hline Fais (KN) & 343.1757 & 312.1698 & 332.3883 & 323.0553 \\
\hline F1 (KN) & 317.0859 & 281.7465 & 305.3179 & 292.6319 \\
\hline F2 (KN) & 328.4332 & 277.2835 & 316.6652 & 288.1689 \\
\hline F3 (KN) & 344.2122 & 265.4105 & 332.4442 & 276.2959 \\
\hline F4 (KN) & 357.5170 & 236.0129 & 345.7489 & 246.8984 \\
\hline F5 (KN) & 237.4016 & 133.5535 & 225.6335 & 144.4390 \\
\hline
\end{tabular}

Tabla 27. Comparación entre estructuras Rígida y Flexible a nivel de Derivas para sismo DBE en sentido "X". Con Método Espectral y SAP2000.

\begin{tabular}{|c|c|c|c|c|}
\hline BLOQUE I ZE & \multicolumn{4}{|c|}{ DERIVAS DE PISO } \\
\hline $\begin{array}{c}\text { TIPO DE } \\
\text { ESTRUCTURA }\end{array}$ & FLEXIBLE & RÍGIDA & FLEXIBLE & RÍGIDA \\
\hline $\begin{array}{c}\text { SENTIDO } \\
\text { "X" }\end{array}$ & $\begin{array}{c}\text { MODAL } \\
\text { ESPECTRAL }\end{array}$ & $\begin{array}{c}\text { MODAL } \\
\text { ESPECTRAL }\end{array}$ & SAP2000 & SAP2000 \\
\hline Derv ais & 0.0699 & 0.0738 & 0.0661 & 0.0733 \\
\hline Derv 1 & 0.0018 & 0.0009 & 0.0020 & 0.0019 \\
\hline Derv 2 & 0.0017 & 0.0009 & 0.0019 & 0.0018 \\
\hline Derv 3 & 0.0015 & 0.0008 & 0.0016 & 0.0016 \\
\hline Derv 4 & 0.0011 & 0.0007 & 0.0012 & 0.0011 \\
\hline Derv 5 & 0.0007 & 0.0006 & 0.0007 & 0.0007 \\
\hline Derv máx. & 0.0018 & 0.0009 & 0.0020 & 0.0019 \\
\hline
\end{tabular}

Tabla 28. Comparación entre estructuras Rígida y Flexible a nivel de Periodos para sismo MCE en sentido "X". Con Método Espectral y SAP2000.

\begin{tabular}{|c|c|c|c|c|}
\hline BLOQUE I ZE & \multicolumn{4}{|c|}{ PERIODOS } \\
\hline $\begin{array}{c}\text { TIPO DE } \\
\text { ESTRUCTURA }\end{array}$ & FLEXIBLE & RÍGIDA & FLEXIBLE & RÍGIDA \\
\hline $\begin{array}{c}\text { SENTIDO } \\
\text { "X" }\end{array}$ & $\begin{array}{c}\text { MODAL } \\
\text { ESPECTRAL }\end{array}$ & $\begin{array}{c}\text { MODAL } \\
\text { ESPECTRAL }\end{array}$ & SAP2000 & SAP2000 \\
\hline T1 (seg) & 3.3961 & 3.8471 & 3.3417 & 3.7115 \\
\hline T2 (seg) & 3.3783 & 3.5002 & 3.3167 & 3.6057 \\
\hline T3 (seg) & 3.2727 & 3.2210 & 3.0866 & 3.5911 \\
\hline
\end{tabular}


Tabla 29. Comparación entre estructura Rígida y Flexible a nivel de Desplazamientos para sismo MCE sentido "X". Con Método Espectral y SAP2000.

\begin{tabular}{|c|c|c|c|c|}
\hline BLOQUE I ZE & \multicolumn{4}{|c|}{ DESPLAZAMIENTOS } \\
\hline $\begin{array}{c}\text { TIPO DE } \\
\text { ESTRUCTURA }\end{array}$ & FLEXIBLE & RÍGIDA & FLEXIBLE & RÍGIDA \\
\hline $\begin{array}{c}\text { SENTIDO } \\
\text { "X" }\end{array}$ & $\begin{array}{c}\text { MODAL } \\
\text { ESPECTRAL }\end{array}$ & $\begin{array}{c}\text { MODAL } \\
\text { ESPECTRAL }\end{array}$ & SAP2000 & SAP2000 \\
\hline q ais (cm) & 46.9697 & 48.6838 & 44.7183 & 49.4580 \\
\hline q1 (cm) & 47.8933 & 49.1344 & 45.7366 & 49.5705 \\
\hline q2 (cm) & 48.7747 & 49.5586 & 46.7087 & 49.7794 \\
\hline q3 (cm) & 49.5250 & 49.9389 & 47.5363 & 50.0157 \\
\hline q4 (cm) & 50.0534 & 50.2702 & 48.1189 & 50.2569 \\
\hline q5 (cm) & 50.3891 & 50.5643 & 48.4887 & 50.4855 \\
\hline
\end{tabular}

Tabla 30. Comparación entre estructuras Rígida y Flexible a nivel de Fuerzas para sismo MCE en sentido "X". Con Método Espectral y SAP2000.

\begin{tabular}{|c|c|c|c|c|}
\hline BLOQUE I ZE & \multicolumn{4}{|c|}{ FUERZAS LATERALES } \\
\hline $\begin{array}{c}\text { TIPO DE } \\
\text { ESTRUCTURA }\end{array}$ & FLEXIBLE & RÍGIDA & FLEXIBLE & RÍGIDA \\
\hline $\begin{array}{c}\text { SENTIDO } \\
\text { "X" }\end{array}$ & $\begin{array}{c}\text { MODAL } \\
\text { ESPECTRAL }\end{array}$ & $\begin{array}{c}\text { MODAL } \\
\text { ESPECTRAL }\end{array}$ & SAP2000 & SAP2000 \\
\hline Fais (KN) & 512.1402 & 443.5757 & 491.5461 & 458.2857 \\
\hline F1 (KN) & 470.9373 & 393.0643 & 450.3433 & 407.7744 \\
\hline F2 (KN) & 481.3148 & 376.6459 & 460.7207 & 391.3560 \\
\hline F3 (KN) & 495.4355 & 346.1951 & 474.8414 & 360.9052 \\
\hline F4 (KN) & 506.5621 & 287.9110 & 485.9681 & 302.6210 \\
\hline F5 (KN) & 324.5704 & 146.7837 & 303.9763 & 161.4938 \\
\hline
\end{tabular}

Tabla 31. Comparación entre estructuras Rígida y Flexible a nivel de Derivas para sismo MCE en sentido "X". Con Método Espectral y SAP2000.

\begin{tabular}{|c|c|c|c|c|}
\hline BLOQUE I ZE & \multicolumn{4}{|c|}{ DERIVAS DE PISO } \\
\hline $\begin{array}{c}\text { TIPO DE } \\
\text { ESTRUCTURA }\end{array}$ & FLEXIBLE & RÍGIDA & FLEXIBLE & RÍGIDA \\
\hline $\begin{array}{c}\text { SENTIDO } \\
\text { "X" }\end{array}$ & $\begin{array}{c}\text { MODAL } \\
\text { ESPECTRAL }\end{array}$ & $\begin{array}{c}\text { MODAL } \\
\text { ESPECTRAL }\end{array}$ & SAP2000 & SAP2000 \\
\hline Derv ais & 0.1342 & 0.1391 & 0.1278 & 0.1413 \\
\hline Derv 1 & 0.0026 & 0.0013 & 0.0029 & 0.0014 \\
\hline Derv 2 & 0.0025 & 0.0012 & 0.0028 & 0.0013 \\
\hline Derv 3 & 0.0021 & 0.0011 & 0.0024 & 0.0012 \\
\hline Derv 4 & 0.0015 & 0.0009 & 0.0017 & 0.0010 \\
\hline Derv 5 & 0.0010 & 0.0008 & 0.0011 & 0.0009 \\
\hline Derv máx. & 0.0026 & 0.0013 & 0.0029 & 0.0014 \\
\hline
\end{tabular}


En las figuras 20 y 21 se presenta los desplazamientos y derivas de piso correspondientes al Método de Superposición Modal, evaluado en el centro de masas para la estructura con y sin muros de corte en sentido " $X$ ". En la curva correspondiente a la estructura flexible existe mayor desplazamiento en relación con la estructura rígida para los dos sismos DBE y MCE; lo mismo sucede con el análisis de derivas para cada estructura.
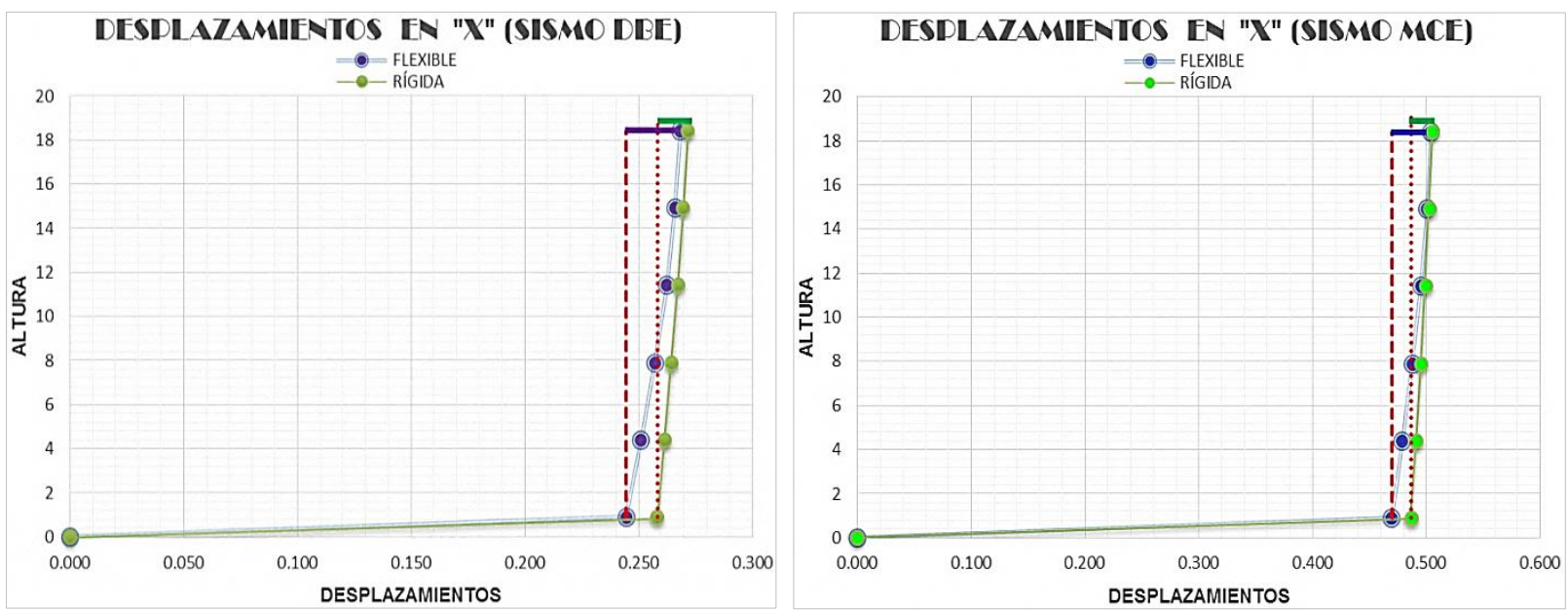

Figura 20. Comparación de Desplazamientos laterales en el centro de masas para sismo DBE y MCE sentido "X". Considerado estructura con y sin muros de corte.
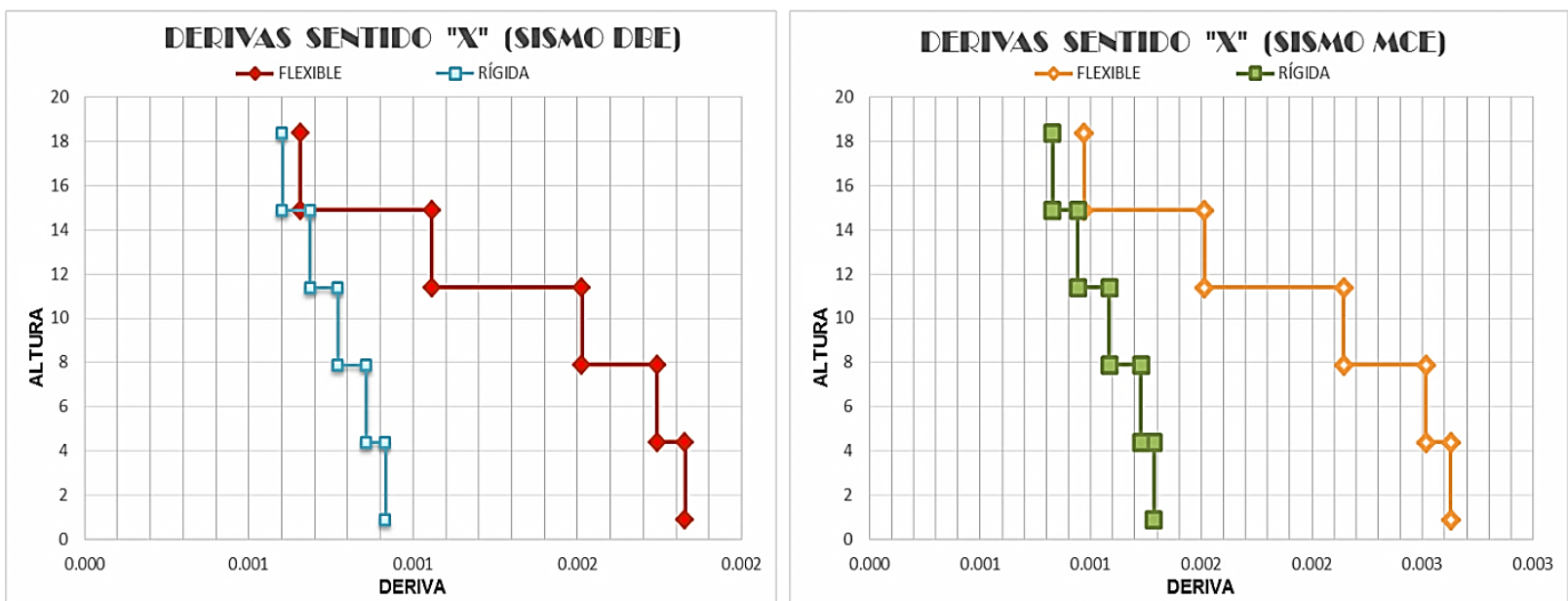

Figura 21. Comparación de Derivas de piso en el centro de masas para sismo DBE y MCE sentido "X". Considerado estructura con y sin muros de corte.

\subsubsection{Resultados comparativos del análisis sísmico en sentido $\mathrm{Y}$}

Con los resultados indicados para el sentido de análisis "X", se establece que la estructura aislada con y sin muros de corte tiene un mejor comportamiento en ésta dirección, dando lugar a que "Y" sea el sentido más crítico para este caso de aplicación. 
Tabla 32. Comparación entre estructuras Rígida y Flexible a nivel de Desplazamientos para sismo DBE sentido "Y". Con Método Espectral y SAP2000.

\begin{tabular}{|c|c|c|c|c|}
\hline BLOQUE I ZE & \multicolumn{4}{|c|}{ DESPLAZAMIENTOS } \\
\hline $\begin{array}{c}\text { TIPO DE } \\
\text { ESTRUCTURA }\end{array}$ & FLEXIBLE & RÍGIDA & FLEXIBLE & RÍGIDA \\
\hline $\begin{array}{c}\text { SENTIDO } \\
\text { "Y" }\end{array}$ & $\begin{array}{c}\text { MODAL } \\
\text { ESPECTRAL }\end{array}$ & $\begin{array}{c}\text { MODAL } \\
\text { ESPECTRAL }\end{array}$ & SAP2000 & SAP2000 \\
\hline q ais (cm) & 24.6267 & 25.7214 & 23.3350 & 25.9696 \\
\hline q1 (cm) & 25.1060 & 26.1172 & 23.8357 & 26.0410 \\
\hline q2 (cm) & 25.5635 & 26.5004 & 24.3146 & 26.1837 \\
\hline q3 (cm) & 25.9518 & 26.8613 & 24.7219 & 26.3516 \\
\hline q4 (cm) & 26.2182 & 27.1955 & 25.0005 & 26.5259 \\
\hline q5 (cm) & 26.3778 & 27.5055 & 25.1656 & 26.6937 \\
\hline
\end{tabular}

Tabla 33. Comparación entre estructuras Rígida y Flexible a nivel de Fuerzas para sismo DBE en sentido "Y". Con Método Espectral y SAP2000.

\begin{tabular}{|c|c|c|c|c|}
\hline BLOQUE I ZE & \multicolumn{4}{|c|}{ FUERZAS LATERALES } \\
\hline $\begin{array}{c}\text { TIPO DE } \\
\text { ESTRUCTURA }\end{array}$ & FLEXIBLE & RÍGIDA & FLEXIBLE & RÍGIDA \\
\hline $\begin{array}{c}\text { SENTIDO } \\
\text { "Y" }\end{array}$ & $\begin{array}{c}\text { MODAL } \\
\text { ESPECTRAL }\end{array}$ & $\begin{array}{c}\text { MODAL } \\
\text { ESPECTRAL }\end{array}$ & SAP2000 & SAP2000 \\
\hline Fais (KN) & 440.1649 & 345.2517 & 386.2281 & 356.1372 \\
\hline F1 (KN) & 405.4963 & 317.2948 & 351.5594 & 328.1802 \\
\hline F2 (KN) & 411.6470 & 323.4054 & 357.7102 & 334.2908 \\
\hline F3 (KN) & 418.5706 & 331.8999 & 364.6337 & 342.7854 \\
\hline F4 (KN) & 425.3225 & 342.3852 & 371.3856 & 353.2707 \\
\hline F5 (KN) & 315.3658 & 259.3813 & 261.4290 & 270.2668 \\
\hline
\end{tabular}

Tabla 34. Comparación entre estructuras Rígida y Flexible a nivel de Derivas para sismo DBE en sentido "Y". Con Método Espectral y SAP2000.

\begin{tabular}{|c|c|c|c|c|}
\hline BLOQUE I ZE & \multicolumn{4}{|c|}{ DERIVAS DE PISO } \\
\hline $\begin{array}{c}\text { TIPO DE } \\
\text { ESTRUCTURA }\end{array}$ & FLEXIBLE & RÍGIDA & FLEXIBLE & RÍGIDA \\
\hline $\begin{array}{c}\text { SENTIDO } \\
\text { "Y" }\end{array}$ & $\begin{array}{c}\text { MODAL } \\
\text { ESPECTRAL }\end{array}$ & $\begin{array}{c}\text { MODAL } \\
\text { ESPECTRAL }\end{array}$ & SAP2000 & SAP2000 \\
\hline Derv ais & 0.0704 & 0.0735 & 0.0667 & 0.0742 \\
\hline Derv 1 & 0.0014 & 0.0011 & 0.0014 & 0.0002 \\
\hline Derv 2 & 0.0013 & 0.0011 & 0.0014 & 0.0004 \\
\hline Derv 3 & 0.0011 & 0.0010 & 0.0012 & 0.0005 \\
\hline Derv 4 & 0.0008 & 0.0010 & 0.0008 & 0.0005 \\
\hline Derv 5 & 0.0005 & 0.0009 & 0.0005 & 0.0005 \\
\hline Derv máx. & 0.0014 & 0.0011 & 0.0014 & 0.0002 \\
\hline
\end{tabular}


Tabla 35. Comparación entre estructuras Rígida y Flexible a nivel de Desplazamientos para sismo MCE sentido "Y". Con Método Espectral y SAP2000.

\begin{tabular}{|c|c|c|c|c|}
\hline BLOQUE I ZE & \multicolumn{4}{|c|}{ DESPLAZAMIENTOS } \\
\hline $\begin{array}{c}\text { TIPO DE } \\
\text { ESTRUCTURA }\end{array}$ & FLEXIBLE & RÍGIDA & FLEXIBLE & RÍGIDA \\
\hline $\begin{array}{c}\text { SENTIDO } \\
\text { "Y" }\end{array}$ & $\begin{array}{c}\text { MODAL } \\
\text { ESPECTRAL }\end{array}$ & $\begin{array}{c}\text { MODAL } \\
\text { ESPECTRAL }\end{array}$ & SAP2000 & SAP2000 \\
\hline q ais (cm) & 47.2039 & 49.4472 & 45.0097 & 50.0495 \\
\hline q1 (cm) & 47.8956 & 49.9020 & 45.7330 & 50.1526 \\
\hline q2 (cm) & 48.5557 & 50.3355 & 46.4241 & 50.3586 \\
\hline q3 (cm) & 49.1160 & 50.6725 & 47.0111 & 50.6010 \\
\hline q4 (cm) & 49.5003 & 50.9539 & 47.4122 & 50.8524 \\
\hline q5 (cm) & 49.7306 & 51.1277 & 47.6496 & 51.0945 \\
\hline
\end{tabular}

Tabla 36. Comparación entre estructuras Rígida y Flexible a nivel de Fuerzas para sismo MCE en sentido "Y". Con Método Espectral y SAP2000.

\begin{tabular}{|c|c|c|c|c|}
\hline BLOQUE I ZE & \multicolumn{4}{|c|}{ FUERZAS LATERALES } \\
\hline $\begin{array}{c}\text { TIPO DE } \\
\text { ESTRUCTURA }\end{array}$ & FLEXIBLE & RÍGIDA & FLEXIBLE & RÍGIDA \\
\hline $\begin{array}{c}\text { SENTIDO } \\
\text { "Y" }\end{array}$ & $\begin{array}{c}\text { MODAL } \\
\text { ESPECTRAL }\end{array}$ & $\begin{array}{c}\text { MODAL } \\
\text { ESPECTRAL }\end{array}$ & SAP2000 & SAP2000 \\
\hline Fais (KN) & 527.7838 & 513.9711 & 503.2671 & 524.8565 \\
\hline F1 (KN) & 483.5998 & 470.5814 & 459.0830 & 481.4668 \\
\hline F2 (KN) & 489.4269 & 474.5815 & 464.9101 & 485.4670 \\
\hline F3 (KN) & 497.6145 & 478.9445 & 473.0978 & 489.8300 \\
\hline F4 (KN) & 506.7936 & 484.9560 & 482.2768 & 495.8415 \\
\hline F5 (KN) & 376.8852 & 359.0929 & 352.3685 & 369.9784 \\
\hline
\end{tabular}

Tabla 37. Comparación entre estructuras Rígida y Flexible a nivel de Derivas para sismo MCE en sentido "Y". Con Método Espectral y SAP2000.

\begin{tabular}{|c|c|c|c|c|}
\hline BLOQUE I ZE & \multicolumn{4}{|c|}{ DERIVAS DE PISO } \\
\hline $\begin{array}{c}\text { TIPO DE } \\
\text { ESTRUCTURA }\end{array}$ & FLEXIBLE & RÍGIDA & FLEXIBLE & RÍGIDA \\
\hline $\begin{array}{c}\text { SENTIDO } \\
\text { "Y" }\end{array}$ & $\begin{array}{c}\text { MODAL } \\
\text { ESPECTRAL }\end{array}$ & $\begin{array}{c}\text { MODAL } \\
\text { ESPECTRAL }\end{array}$ & SAP2000 & SAP2000 \\
\hline Derv ais & 0.1349 & 0.1413 & 0.1286 & 0.1430 \\
\hline Derv 1 & 0.0020 & 0.0013 & 0.0021 & 0.0003 \\
\hline Derv 2 & 0.0019 & 0.0013 & 0.0020 & 0.0006 \\
\hline Derv 3 & 0.0016 & 0.0009 & 0.0017 & 0.0007 \\
\hline Derv 4 & 0.0011 & 0.0008 & 0.0011 & 0.0007 \\
\hline Derv 5 & 0.0007 & 0.0005 & 0.0007 & 0.0007 \\
\hline Derv máx. & 0.0020 & 0.0013 & 0.0021 & 0.0003 \\
\hline
\end{tabular}




\section{COMENTARIOS Y CONCLUSIONES}

Se ha presentado el marco teórico del Método Simplificado Lineal, Método Espectral y el ingreso de aisladores de triple péndulo de fricción FPT al SAP2000 para un Análisis Lineal de estructuras aisladas con y sin muros de corte. En el primer método se empleó para los aisladores la curva constitutiva del ASCE 7-10 modelo bilineal y en el segundo la curva constitutiva de tres fases.

El análisis sísmico de la estructura rígida y la flexible se lo realizó en sentido " $X$ " y "Y" para dos sismos: DBE y MCE; para el caso del software estructural SAP2000 se analizó de igual forma, es decir cargando los dos espectros sísmicos en formato txt.

A nivel de fuerzas el Método Simplificado Lineal propuesto por el ASCE 710 reporta mayores valores en comparación con el Método de Superposición Modal para la estructura rígida.

Es importante mencionar que se aplicó al programa SAP2000 un Análisis Lineal bajo las mismas condiciones como fue resuelto con el Método Espectral utilizando CEINCI-LAB bajo el criterio de combinación modal CQC propuesto por Chopra (2001), cumpliendo adecuadamente la validación de resultados entre los dos programas sísmicos estructurales.

Por otra parte, se presentó los resultados obtenidos mediante la comparación entre las dos estructuras rígida y flexible concluyendo que, a nivel de desplazamientos se obtuvo el mayor valor con la estructura sin muros de corte de $2.38 \mathrm{~cm}$ para el último piso con respecto a la deformación del aislador y $1.34 \mathrm{~cm}$ para la estructura rígida con el sismo DBE, afirmando que la estructura aislada con muros de corte tiene el comportamiento más favorable ante eventos sísmicos con gran magnitud.

De igual forma para el sismo MCE, se muestra la misma relación óptima para la estructura rígida. Para éste sismo la deformación en el último piso es de $3.42 \mathrm{~cm}$ para la estructura flexible y $1.88 \mathrm{~cm}$ para la rígida, es decir la estructura aislada con muros de corte se mueve como cuerpo rígido, claramente se observa como mejora considerablemente el comportamiento sismo resistente al tener estructuras rígidas de este tipo. 


\section{REFERENCIAS}

1. American Society of Civil Engineers (ASCE 7-10), Code 7: Minimum Design Loads for Buildings and Other Structures. (2010), 608 p.

2. Constantinou M., Aguiar R., Morales E., Caiza P., (2016), "Desempeño de aislador FPT8833/12-12/8-5 en el análisis sísmico del Centro de Investigaciones y de Post Grado de la UFA-ESPE", Revista Internacional de Ingeniería de Estructuras, 21, 1, 1-25.

3. Constantinou, M. C, Whittaker, A. S., Kalpakidis, Y., Fenz, D. M. and Warn G. P. (2007), "Performace of Seismic Isolation Hardware under Service and Seismic Loading," Multidisciplinary Center for Earthquake Engineering Research, Technical Report MCEER-07-0012, Buffalo, NY.

4. Chistopupoulus,C. and Filiatraul,A.(2006). Priciples of passive Suplemental Damping and Seismic Isolation. IUSS Press, Instituto Universitario di Studi Superiori di Pavia, Pavia, Pavia,IT.

5. Chopra A. K. (2001), Dynamic of structures: Theory and application's to earthquake engineering, 2nd edn. Prentice Hall: Saddle River New York.

6. EPS (2015), "Production Bearing Test Results for Ecuador University Building (ESPE), Earthquake Protection System, EPS Inc., Mare Island, Vallejo, California.

7. Fadi F., and Constantinou M. (2010), "Evaluation of simplified methods of analysis for structures with triple friction pendulum isolators", Earthquake Engineering and Structural Dynamics, 39, 5-22.

8. Fenz, D. and Constantinou, M.C. (2006), "Behavior of Double Concave FrictionPendulum Bearing," Earthquake Engineering and Structural Dynamics, Vol. 35,No.11, 1403-1424.

9. Fenz D., Constantinou M., (2007), Mechanical behavior of multi-spherical sliding bearings, Technical Report MCEER-08-0007, Multidisciplinary Center for Earthquake Engineering Research.

10. Fenz, D.M. and Constantinou, M.C., (2008), Development, implementation and verification of Dynamic Analysis Models for Multi-Spherical sliding bearings. Report No. MCEER-08-0018, Multidisciplinary Center for Earthquake Engineering Research, Buffalo, NY.

11. McVitty, W.J. and Constantinou, M.C., (2015), Property modification factors for seismic isolators: Design Guidance for Buildings, Technical Report No. MCEER-15-0005, Multidisciplinary Center for Earthquake Engineering Research, State University of New York at Buffalo, Buffalo, NY, 2015.

12. Naeim, C., and Kelly, J.M., (1999), Desing of Seismic Isolation Structures, John Wiley \& Sons Inc. 
13. NEC-SE-DS (2015) Norma Ecuatoriana de la Construcción, Ministerio de Desarrollo Urbano y Vivienda, MIDUVI.

14. Norma Ch2745, (2013), Análisis y diseño de edificios con aislación sísmica, Norma Chilena, Segunda Edición, 88 p., Santiago.

15. Kawamura S., Sugisaki R., Ogura K., Maezawa S., Tanaka S., Yajima A., (2000), "Seismic Isolation retrofit in Japan", 12 World Conferences on Earthquake Engineerin WCEE, 2523, 8p. New Zeland.

16. Retamales, R., Bonelli, P., Boroschek, R. y Carvallo, J., (2015), "Aplicación de sistemas de aislación sísmica en edificaciones de mediana altura", XI Congreso Chileno de Sismología e Ingeniería Sísmica, Paper 184, 9 p, Santiago.

17. Saito, T., liba, M., Morita, K., Azuhata, T., and Inoue N., (2012), "Seismic performance of seismically isolated buidings at the great east Japan earthquake", $12^{\text {th }}$ World Conference on Seismic Isolation, Energy Dissipation and Active Vibration Control of Structures, Sochi, Rusia.

18. Zayas, V., (2013), Seismic Isolation Desing for Resilient Building , Base Isolation Systems: Applications, Codes \& Quality Control Tests", Istanbul Technical University.

19. Constantinou, M.C., Whittaker, A.S., Kalpakidis, Y., Fenz, D.M. and Warn, G.P. (2007a), "Performance of Seismic Isolation Hardware under Service and Seismic Loading," Report No. MCEER-07-0012, Multidisciplinary Center for Earthquake Engineering Research, Buffalo, NY.

20. Ismail $M$, Rodellar J, Ikhouane $F$ (2010). An innovative isolation device for aseismic design. Eng. Struct., 32: 1168-1183.

21. Micheli I, Cardini S, Colaiuda A, Turroni $P$ (2004). Investigation upon the dynamic structural response of a nuclear plant on aseismic isolating devices. Nuclear Eng. Des., 228: 319-343.

22. Nicholas R. Marrs (2013). Seismic performance comparison of a fixed-base versus a base-isolated office building. p.196.

23. El-Bayoumi K., (2015), "Modelling of Triple Friction Pendulum Bearing in SAP2000", International Journal of Advances in Engineering \& Technology, ISSN: 22311963, Mansoura, Egypt. February 2015. 